\title{
Mission Functionality for Deflecting Earth-Crossing Asteroids/Comets6
}

\author{
SANG-YOUNG PARK \\ Swales Aerospace \\ DANIEL D. MAZANEK \\ NASA Langley Research Center
}

\section{Introduction}

An infrequent but significant hazard to life and property due to impacting asteroids and comets exists. Earth-approaching asteroids and comets also represent a significant resource for commercial exploitation, space exploration, and scientific research. The impact problem and those planetary bodies that could be a threat have been discussed in great depth in a wide range of publications. A popular planetary defense method is the deflection of asteroids and comets on a collision orbit with the Earth by changing their orbital velocities. It is fundamental to estimate required changes in the orbital velocities of dangerous objects to avoid a collision.

A detailed optimization problem is formulated to calculate optimal impulses for deflecting Earthcrossing asteroids/comets, using nonlinear programming. The constrained optimization problem is based on a three-dimensional patched conic method to include the Earth's gravitational effects and the asteroid/ comet's orbital inclination. The magnitudes and impulse angles of optimal impulse change in velocity $(\Delta \mathrm{V})$ are accurately computed at various points on the asteroid/comet's orbit to provide a given target separation distance. Based on these change in velocity analyses, we can establish the approximate cost and build strategies to prevent possible catastrophe due to the objects.

\section{Background}

The optimal $\Delta \mathrm{V}$ and deflection strategy are dependent on the size and the orbital elements of the asteroid/comet, as well as the amount of warning time. Assuming a linear approximation between orbital energy and velocity increment, a velocity increment of about $1 \mathrm{~cm} / \mathrm{s}$ is required to deflect an asteroid by a distance equal to 1 Earth radius for the order of a decade ahead of an impact (ref. 1). Using Keplerian motion and perpendicular impulse direction, both kinetic-energy deflection and nuclear-explosive deflection are treated in reference 2. A method in reference 3 shows the instantaneous correction of asteroid velocity as a result of a spacecraft's collision with an asteroid at perihelion only. In reference 4 the use of a nuclear explosive is discussed and it is concluded that a nuclear device having a yield of about $1 \mathrm{Mt}$ may be required to deflect an object with a $0.3-\mathrm{km}$ radius at a distance of 1 astronomical unit (au). Direct spacecraft impacts on Earth-crossing objects (ECOs) can provide kinetic energy to suitably disrupt up to about $0.1-\mathrm{km}$ stony asteroids and $0.3-\mathrm{km}$ ice comets (ref. 5). One clear conclusion from these simplified analyses is that early detection gives longer reaction time, and asteroid/comet interceptions far from Earth are much easier and more desirable than interceptions near Earth because small deflections far away will produce a greater miss distance at the Earth. It is important to consider interception several orbital periods in advance as well as interceptions close to the Earth because both of these situations could be encountered depending on the size and orbital characteristics of an asteroid/comet.

Recently, the astrodynamical optimization problem was formulated for Earth-crossing asteroids (ECAs) and presented accurate impulse angles as well as impulse magnitude according to impulse time

6Chapter nomenclature available in chapter notes, p. 217. 
based on a two-dimensional, two-body analysis (ref. 6). The minimum impulse change in velocity $(\Delta \mathrm{V})$ requirement is not a monotonically decreasing function of warning time; rather, there is a finer structure associated with the orbital period of the colliding asteroid. Thus, the "optimal time" for application of $\Delta \mathrm{V}$ is the earliest possible perihelion for warning times greater than 1 orbital period (ref. 6). However, this research has simplified the problem by assuming two-body orbital mechanics between the Sun and an ECA. This assumption neglects perturbations due to the Earth's gravity. While these perturbations may not be present until the terminal phase of the impact scenario, they affect both long and short warning time analyses. Further research refines the heliocentric two-body analysis presented in reference 6 by including the gravitational effect of the Earth (ref. 7). The minimum $\Delta \mathrm{V}$ is increased due to gravitational effects of the Earth, and effects on the minimum $\Delta \mathrm{V}$ are sensitive to orbital elements of a target asteroid because the impact parameter is dependent upon those orbital elements. Gravitational effects of Earth have the strongest influence on minimum $\Delta \mathrm{V}$ calculations for deflecting ECAs in nearly circular heliocentric orbits around the Earth. Hence, reference 7 concludes that the problem of deflecting ECAs should include the gravitational effects of Earth; the results in reference 7 followed from two-dimensional analysis. A near-optimal analysis for three-dimensional deflecting problems is described by using a system transition matrix (ref. 8). The study in reference 8 does not include Earth's gravitational effects and is a first-order approximation because of the nature of the system transition matrix used. To have insights in deflecting Earth-approaching asteroids and comets with nonzero inclination, this paper expands the works of reference 7 by formulating an optimal deflection problem in three-dimension that also includes the Earth's gravitational effects. The analysis of the impact deflection problem in this paper is based on a three-dimensional patched conic method. The analysis centers on how impulses applied to an asteroid at various points on the asteroid's orbit affect the outcome when there is a presumption of collision otherwise. The analysis tool presented can be utilized in determining an accurate estimate for optimizing the time and position of intercepting the asteroid and comet for impact deflection.

Deflection or orbit modification methods will be dependent on the size and composition of asteroids/ comets and the amount of warning time available. There are several deflection schemes such as the propulsive mode, kinetic deflection, nuclear detonation, and laser ablation. A study of spacecraft vertical landing on an asteroid indicates the possibility of giving a significant boost to an asteroid by means of a buried explosion of practical yield (refs. 9 and 10) and a high-thrust or low-thrust engine. Because every kilogram of propellant landed on an asteroid requires thousands of times more mass to lift it from the Earth and deliver it to the object, landing propellant on the asteroid in order to provide working mass is extremely inefficient (ref. 11). However, this strategy can easily control motion of an asteroid/comet because it has many opportunities to choose the direction and time of impulse application (ref. 3). Kinetic deflection and nuclear detonation demand much less "cost" than the propulsive mode and, consequently, require much less initial mass for deflection missions (ref. 12). A high-thrust, laser ablation technique should be seriously considered for use in deflecting or disrupting threatening celestial objects because these schemes appear both practical and possible. In this paper, we briefly discuss the potential of each strategy to deflect ECOs in conjunction with several future spacecraft concepts.

\section{Three-Dimensional Optimality Problem for $\Delta V$ Analysis}

Given an ECO and an established Earth collision, the problem is to minimize the impulse change in velocity $(\Delta \mathrm{V})$ required to deflect the ECO in such a manner as to miss the Earth by a minimum target miss distance (see fig. 1). The performance index is defined by the magnitude of the required deflection velocity:

$$
\mathrm{J}(\mathbf{u})=\sqrt{\Delta \mathrm{V}_{\mathrm{T}}^{2}+\Delta \mathrm{V}_{\mathrm{N}}^{2}+\Delta \mathrm{V}_{\mathrm{W}}^{2}}
$$


TNW coordinate system

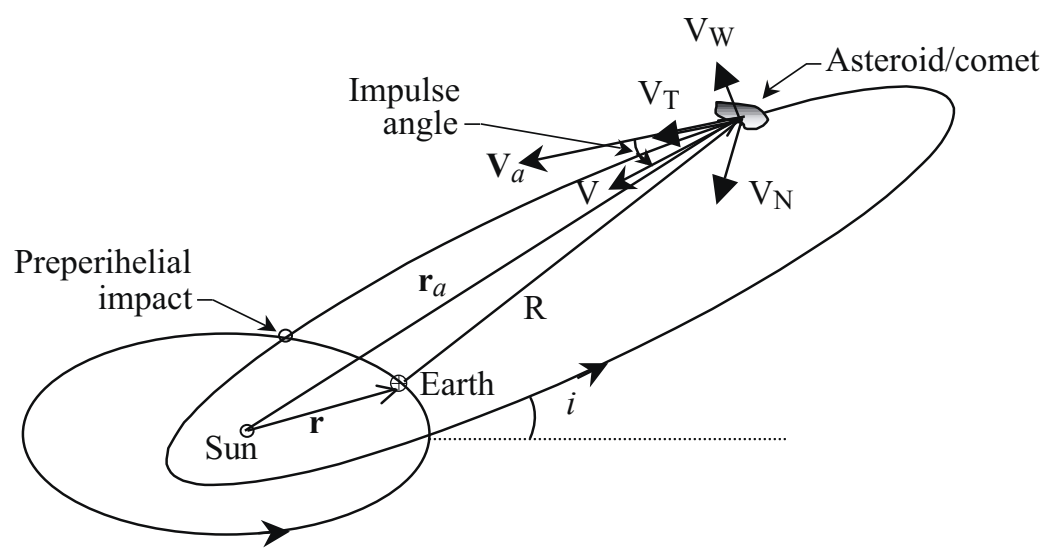

Figure 1. Geometry of Earth-crossing asteroid/comet (not to scale) with TNW coordinate system.

where $\Delta \mathrm{V}_{\mathrm{T}}$ is the velocity increment aligned with the ECO velocity, $\Delta \mathrm{V}_{\mathrm{N}}$ is the velocity increment normal to $\Delta \mathrm{V}_{\mathrm{T}}$ in the $\mathrm{ECO}$ orbital plane, and $\Delta \mathrm{V}_{\mathrm{W}}$ is the velocity increment normal to the $\mathrm{ECO}$ orbital plane. The impulse controls, $\mathbf{u}$, are $\Delta \mathrm{V}_{\mathrm{T}}, \Delta \mathrm{V}_{\mathrm{N}}$, and $\Delta \mathrm{V}_{\mathrm{W}}$, which effectively give the magnitude $\left(|\Delta \mathrm{V}|=\sqrt{\Delta \mathrm{V}_{\mathrm{T}}^{2}+\Delta \mathrm{V}_{\mathrm{N}}^{2}+\Delta \mathrm{V}_{\mathrm{W}}^{2}}\right)$ and angle of the deflection velocity. The geometry of an ECA or comet with the "TNW" coordinate system is shown in figure 1. The calculation is always done to move the ECO's trajectory from crossing the Earth's orbit at the Earth's center. This problem is subject to the heliocentric two-body equation outside the Earth's sphere-of-influence (SOI) and geocentric two-body equations inside the SOI. Thus, the analysis of the impact deflection problem is based on a threedimensional patched conic method. It is assumed that an ECO is influenced by the gravitational field of the Earth only when it is within the Earth's SOI. Beyond the SOI, the object is considered to be affected only by the Sun's gravitation. In the case of an impact scenario, the ECO begins in an elliptical orbit about the Sun. Once within the Earth's SOI, the object's motion is described by two-body orbit equations for a hyperbolic orbit about the Earth. The radius of the Earth's SOI is about $9.31 \times 10^{5} \mathrm{~km}$, or $0.00621 \mathrm{au}$. In figure 2, the approaching distance and a hyperbolic trajectory (ref. 13) are illustrated, and the geometry is still valuable for three-dimensional analysis. Under this framework, the constraints for the optimization problem can be described in terms of the terminal boundary conditions at the time $\left(\mathrm{t}_{\mathrm{f}}\right)$ when the ECO intersects the Earth's SOI:

$$
\begin{gathered}
\mathrm{R}-\mathrm{R}_{\mathrm{SOI}}=0 \\
b-b_{\mathrm{i}}=0 \\
\dot{\mathrm{R}}<0
\end{gathered}
$$

where $\mathrm{R}$ is the distance between Earth and the ECO, $\mathrm{R}_{\mathrm{SOI}}$ is the radius of Earth's $\mathrm{SOI}, \dot{\mathrm{R}}$ is the time derivative of $\mathrm{R}, b$ is the approach distance of the ECO, and $b_{\mathrm{i}}$ is an impact parameter of the Earth. The constraints above mean that the approach distance $(b)$ of the approaching ECO must be equal to the impact parameter $\left(b_{\mathrm{i}}\right)$ at the edge of the Earth's SOI in order to deflect the ECO by a minimum target 


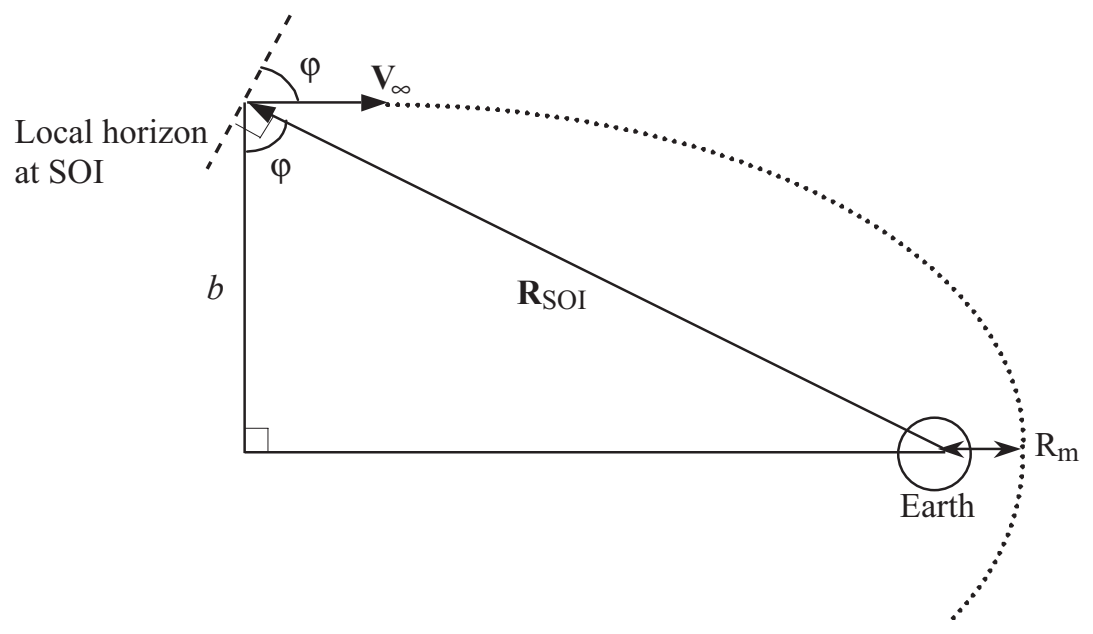

Figure 2. Approach distance, $b$, at SOI of Earth (not to scale).

miss distance $\mathrm{R}_{\mathrm{m}}$. If the approach distance is less than the impact parameter, the ECO can be inside the target miss distance. When we set $\mathrm{R}_{\mathrm{m}}=1$ Earth radius, and $b<b_{\mathrm{i}}$, the ECO will collide with Earth. Note that if $b=b_{\mathrm{i}}$, there will be a surface graze and the case is not considered as a collision in our analysis. If the approach distance is greater than the impact parameter, the impulse will not be a minimum value, forcing the ECO to miss the Earth by $\mathrm{R}_{\mathrm{m}}$. The variable $\mathrm{R}$ is given by

$$
\mathrm{R}=\sqrt{\mathbf{r}_{\oplus} \cdot \mathbf{r}_{\oplus}+\mathbf{r}_{a} \cdot \mathbf{r}_{a}-2 \mathbf{r}_{\oplus} \cdot \mathbf{r}_{a}}
$$

Then, we have

$$
\dot{\mathrm{R}}=\left[\mathbf{r}_{\oplus} \cdot \mathbf{V}_{\oplus}+\mathbf{r}_{a} \cdot \mathbf{V}_{a}-\left(\mathbf{r}_{\oplus} \cdot \mathbf{V}_{a}+\mathbf{r}_{a} \cdot \mathbf{V}_{\oplus}\right)\right] / \mathrm{R}
$$

where $\mathbf{r}_{\oplus}$ is the radius vector from the Sun to the Earth, $\mathbf{r}_{a}$ is the radius vector from the Sun to the ECO, $\mathbf{V}_{a}$ is the velocity of the ECO with respect to the Sun, and $\mathbf{V}_{\oplus}$ is the velocity of the Earth with respect to the Sun. In the heliocentric system, $\mathbf{V}_{\infty}$ is the vector difference at $\mathbf{R}_{\mathrm{SOI}}$ between $\mathbf{V}_{a}$ and $\mathbf{V}_{\oplus}$, so that

$$
\mathbf{V}_{\infty}=\mathbf{V}_{a}-\mathbf{V}_{\oplus}
$$

The vector $\mathbf{V}_{\infty}$ is described by its magnitude $V_{\infty}$ and an elevation angle, $\varphi$. From figure 2 , the elevation angle is given by

$$
\cos \left(\varphi+90^{\circ}\right)=\mathbf{V}_{\infty} \cdot \mathbf{R}_{\mathrm{SOI}} / \mathrm{V}_{\infty} \mathrm{R}_{\mathrm{SOI}}
$$

and the approach distance is obtained from

$$
b=\mathrm{R}_{\mathrm{SOI}} \cos \varphi
$$

The impact parameter, $b_{\mathrm{i}}$, yields the expression

$$
b_{\mathrm{i}}=\mathrm{R}_{\mathrm{m}} \sqrt{1+\frac{\mathrm{V}_{\mathrm{esc}}^{2}}{\mathrm{~V}_{\infty}^{2}}}
$$


where

$$
\mathrm{V}_{\mathrm{esc}}=\sqrt{2 \mu_{\oplus} / \mathrm{R}_{\mathrm{m}}}
$$

$\mathrm{V}_{\text {esc }}$ is the escape velocity at $\mathrm{R}_{\mathrm{m}}$, and $\mu_{\oplus}$ is the gravitational constant of the Earth.

As noted in reference 6, closed-form solutions cannot be obtained for this optimization problem. To solve the heliocentric three-dimensional two-body motion, the method of Lagrangian coefficients (ref. 14) is used for describing $\mathbf{r}(\mathrm{t})$ and $\mathbf{V}(\mathrm{t})$ in terms of initial position vector, $\mathbf{r}\left(\mathrm{t}_{0}\right)$, and velocity vector, $\mathbf{V}\left(\mathrm{t}_{0}\right)$, in the inertial coordinate frame. At impulse time, $\mathrm{t}_{\text {impulse }}$, the original orbit of an ECO is perturbed by an impulse; hence,

$$
\begin{gathered}
\mathbf{r}\left(\mathrm{t}_{\text {impulse }}\right)=\mathbf{r}_{0}\left(\mathrm{t}_{\text {impulse }}\right) \\
\mathbf{V}\left(\mathrm{t}_{\text {impulse }}\right)=\mathbf{V}_{0}\left(\mathrm{t}_{\text {impulse }}\right)+\Delta \mathbf{V}_{\mathrm{XYZ}}\left(\mathrm{t}_{\text {impulse }}\right)
\end{gathered}
$$

$\Delta \mathbf{V}_{\mathrm{XYZ}}$ can be expressed in the inertial coordinate system $(\mathrm{X}, \mathrm{Y}, \mathrm{Z})$ as follows:

$$
\Delta \mathbf{V}_{\mathrm{XYZ}}=\mathrm{R}_{3}(-\Omega) \mathrm{R}_{1}(-i) \mathrm{R}_{3}(-\omega) \mathrm{R}_{3}(-\alpha) \Delta \mathbf{V}_{\mathrm{TNW}}
$$

where $\mathrm{R}_{3}(-\Omega), \mathrm{R}_{1}(-i), \mathrm{R}_{3}(-\omega)$, and $\mathrm{R}_{3}(-\alpha)$ are rotation matrices about the longitude of ascending node $(\Omega)$, the inclination $(i)$, the argument of periapsis $(\omega)$ for ECO, and the angle $(\alpha)$ between ECO perifocal coordinate and TNW system, respectively. Using $\mathbf{r}\left(\mathrm{t}_{\text {impulse }}\right)$ and $\mathbf{V}\left(\mathrm{t}_{\text {impulse }}\right)$ as initial conditions for the perturbed orbit, $\mathbf{r}\left(\mathrm{t}_{\mathrm{f}}\right)$ and $\mathbf{V}\left(\mathrm{t}_{\mathrm{f}}\right)$ are computed at the time when $\mathrm{R}=\mathrm{R}_{\mathrm{SOI}}, \mathrm{t}_{\mathrm{f}}$.

Nonlinear programming (NLP) problems require determining $\mathbf{x} \in \mathrm{R}^{\mathrm{n}}$, with $\mathbf{x}$ as the state vector, that minimize the scalar objective function $\mathrm{J}(\mathbf{u})$ subject to the equality constraints, $\mathrm{C}_{\mathrm{i}}(\mathbf{x})=0$, and the inequality constraints, $\mathrm{C}_{\mathrm{j}}(\mathbf{x}) \leq 0$. It is assumed that the objective function is continuously differentiable through second order. The parameters needed to obtain the constraint equations can be calculated using the relationship between the orbital elements and the position and velocity vectors. For this deflection problem, the free parameters are $\Delta \mathrm{V}_{\mathrm{T}}, \Delta \mathrm{V}_{\mathrm{N}}, \Delta \mathrm{V}_{\mathrm{W}}$, and $\mathrm{t}_{\mathrm{f}}$, equations (2) and (3) are the equality constraints, $\mathrm{C}_{1}$ and $\mathrm{C}_{2}$, and equation (4) is the inequality constraint, $\mathrm{C}_{3}$. The parameter $\mathrm{t}_{\mathrm{f}}$ describes the time at which the constraints given by equations (2) through (4) are satisfied. The problem of minimizing the $\Delta \mathrm{V}$ required for deflecting ECOs can now be cast in terms of a standard NLP problem. This formulation is also applicable to any Earth-approaching objects.

\section{Numerical Results of $\Delta \mathbf{V}$ Analysis}

This analysis indicates the optimal impulses applied on an ECO at various points on the object's orbit to provide the miss distance equal to $\mathrm{R}_{\mathrm{m}}$ when there is otherwise a presumption of collision at Earth's center. The NLP formulated in the previous section was solved by using the MATLAB ${ }^{\mathrm{TM}}$ optimization tool box (ref. 15). In the discussions to follow, we will use the term impulse time to specifically mean either $\left(t_{\text {impact }}-t_{\text {impulse }}\right)$ or its absolute value. Here $t_{\text {impact }}$ denotes the time at collision. Although the impact time is quite close to $t_{f}$, it is not the same because $t_{f}$ is the time when $R=R_{S O I}$. Also, because $t_{\text {impact }}$ and $t_{\text {impulse }}$ are not independent quantities, we choose $t_{\text {impact }}=0$. This initialization has the advantage of interpreting $t_{\text {impulse }}$ as the time interval prior to impact if no action (i.e., $\Delta \mathrm{V}$ maneuver) is undertaken. Also, it is apparent that we must have a warning time (i.e., the time interval between detection and collision) greater than the impulse time. The impulse time provides a crude measure of the 
warning time. The gravitational effects of the Earth are considered by using the three-dimensional optimization problem described previously. The minimum required impulses for the deflecting problem have a targeted distance of 1 Earth radius. The solutions to the deflection problems represent impulse vectors that can be described by the magnitude of the minimum impulse and the optimal impulse angle. The impulse angle is described in the ECO's orbital plane and is defined as the angle from the ECO's original velocity vector to the impulse vector toward the Sun-ECO line, $\mathbf{r}_{a}$ (see fig. 1). As reference 8 indicates, most numerical simulations show that the velocity increment $\left(\Delta \mathrm{V}_{\mathrm{W}}\right)$ normal to the ECO's orbital plane is insignificant and ignored for all cases in these analyses. Thus, the magnitude of impulse consists of only $\Delta \mathrm{V}_{\mathrm{T}}$ and $\Delta \mathrm{V}_{\mathrm{N}}$. For any given impulse time, the problem has two solutions for the optimal impulse angle separated by $180^{\circ}$ while keeping the same magnitude of impulse (ref. 6). In this section, only one solution for the optimal angle will be mentioned.

There are two categories of impact scenarios: one (impact before perihelion, or preperihelial impact) is that an impact occurs before the ECO sweeps its perihelion (fig. 1), the other (impact after perihelion, or postperihelial impact) is that an impact occurs after the ECO passes its perihelion. The minimum impulse requirements for the two impact scenarios are similar but slightly different. The differences are caused by the varied geometric positions of the impulse point with respect to the Sun. Each impact scenario has two subscenarios: an impact occurs either at the ascending node or at the descending node of an ECO. These two subscenarios have identical impulse angles and magnitudes because the subscenarios have the same geometric positions of the impulse point. We present results from some $\Delta \mathrm{V}$ analyses while our method is applicable to any ECO deflection mission.

\section{Near-Earth Asteroids}

There are three classes of near-Earth asteroids (NEAs): Atens, Apollos, and Amors. Aten-type asteroids have a semimajor axis smaller than 1 au and an aphelion greater than $0.983 \mathrm{au}$, while Apollo-type asteroids have a semimajor axis greater than 1 au and a perihelion smaller than 1.017 au. Hence, Apollotype and Aten-type asteroids can have Earth-crossing orbits. Amors have orbits that lie completely outside Earth's orbit (perihelial distance between 1.017 and $1.3 \mathrm{au}$ ) but have the potential to be perturbed into Earth-crossing trajectories.

We consider fictitious Apollo-type asteroids with semimajor axis $a=1.5 \mathrm{au}$, eccentricity $e=0.5$, inclination $i=0^{\circ}, 20^{\circ}, 40^{\circ}$, and $60^{\circ}$ first. For this example, the asteroids have orbital periods of approximately 1.84 years, 0.75 au perihelial distance, and 2.25 au aphelial distance. Figures 3 and 4 include the magnitudes and angles of the optimal impulses. The impulse time is normalized to the period of the unperturbed asteroid for ease of interpretation. The abscissa represents the time when impulse is applied (as a fraction of the period of the asteroid) prior to collision. It can also be noticed that the separation between Earth and an asteroid, which can be achieved by an impulse, depends strongly on location of the impulse on the orbit as well as direction of the impulse with respect to the orbital velocity. The required minimum $\Delta \mathrm{V}$ has a cyclic component imposed upon a secular variation that varies inversely with the impulse time. Generally, the minimum $\Delta \mathrm{V}$ for inclined orbits $\left(i>0^{\circ}\right)$ is slightly less than that for planar orbits $\left(i=0^{\circ}\right)$. The case of preperihelial impact has more fluctuated magnitude variations than the case of postperihelial impact. Figure 4 contains a parallel history of the optimal impulse angle with respect to impulse time. It is apparent that the optimal angles for both planar and inclined cases are almost the same if the impulse takes place at more than half an orbital period of the asteroid before impact. There are fluctuations in the optimal impulse angle when the $\Delta \mathrm{V}$ occurs less than half an orbital period before impact. The fluctuations are more for an orbit with a higher inclination than for one with a lower inclination. Generally, postperihelial cases have more fluctuations in impulse angle than preperihelial cases. 


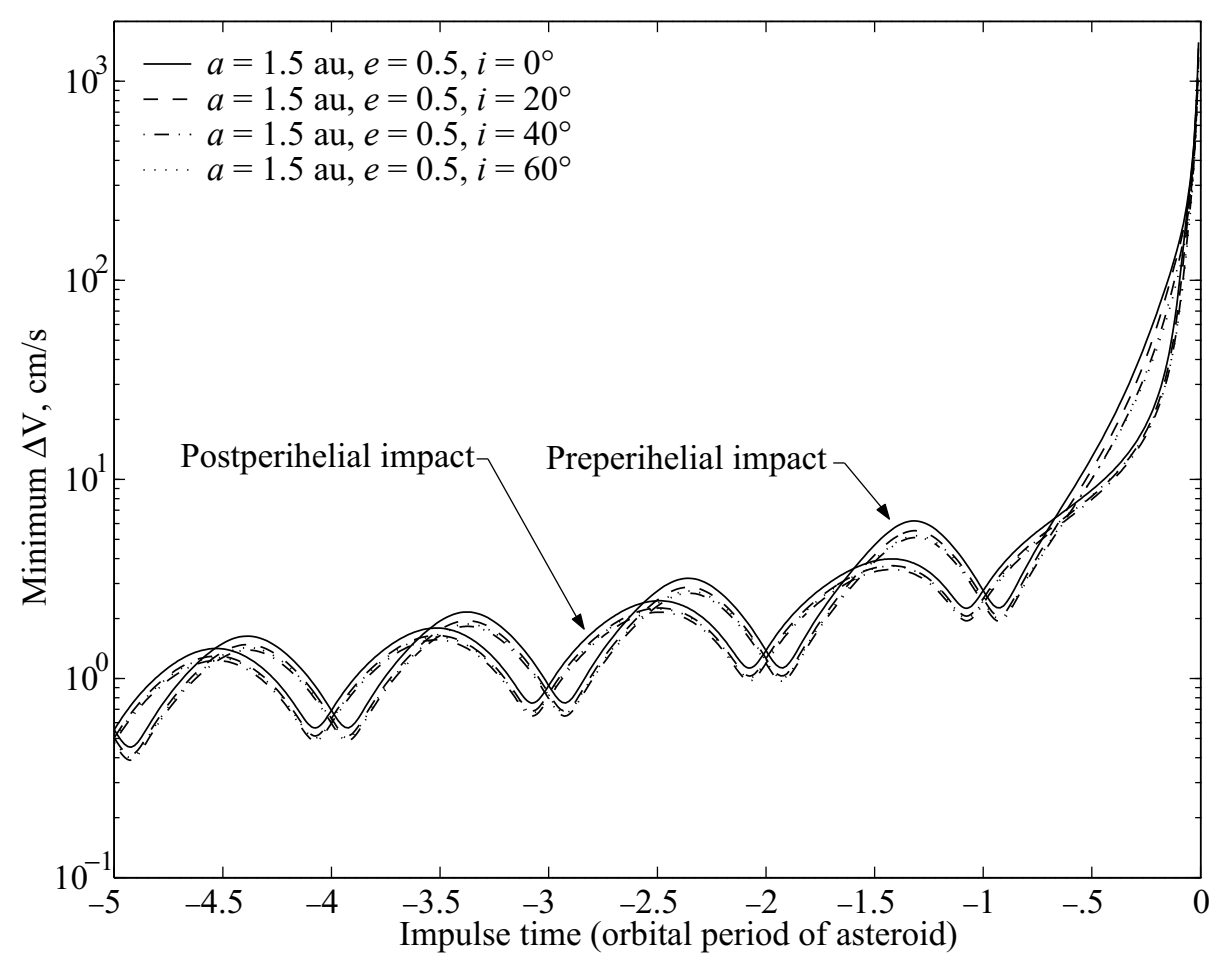

Figure 3. Minimum $\Delta \mathrm{V}$ : Apollo-type asteroids with $a=1.5$ au, $e=0.5, i=0^{\circ}, 20^{\circ}, 40^{\circ}, 60^{\circ}$.

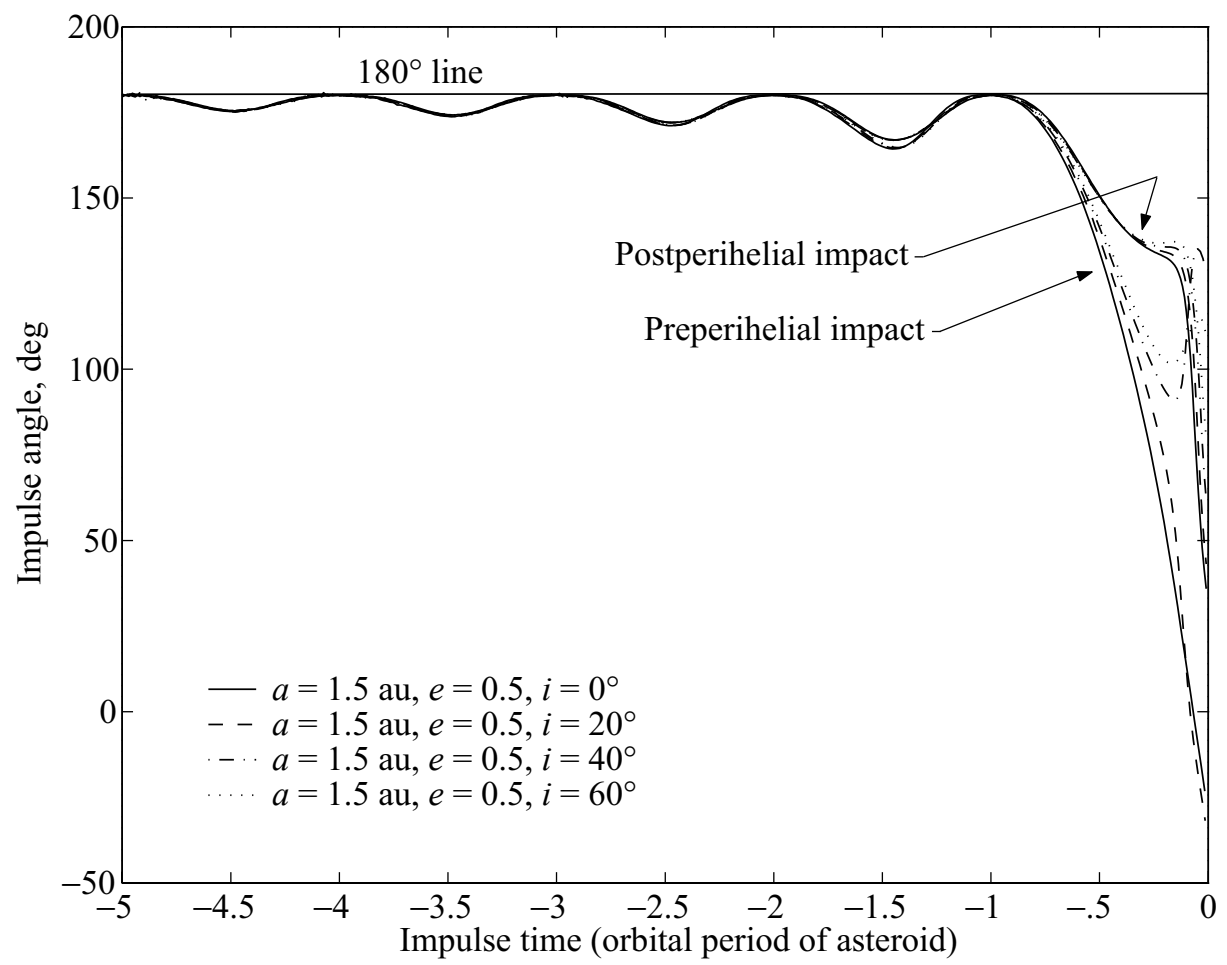

Figure 4. Impulse angle: Apollo-type asteroids with $a=1.5 \mathrm{au}, e=0.5, i=0^{\circ}, 20^{\circ}, 40^{\circ}, 60^{\circ}$. 
Figures 5 and 6 include the magnitudes and angles of the optimal impulses for another set of Apollotype asteroids whose orbital elements are $a=2.0$ au (orbital period $\approx 2.83$ years), $i=20^{\circ}$, and $e=0.6,0.7$, 0.8 , and 0.9 . From these analyses, we know that higher eccentricity yields more fluctuated histories of impulse magnitude and angle. Figures 7 and 8 include the magnitudes and angles of the optimal impulses for a third set of Apollo-type asteroids whose orbital elements are $e=0.7, i=10^{\circ}$, and $a=1.2$ au (orbital period $\approx 1.31$ years), 1.6 au (orbital period $\approx 2.02$ years), 2.0 au (orbital period $\approx 2.83$ years), and 2.4 au (orbital period $\approx 3.72$ years). The minimum $\Delta \mathrm{V}$ decreases with increasing orbital period (i.e., larger semimajor axis) because a longer orbital period yields a longer warning time.

Figures 9 through 14 contain data demonstrating that the same trends are apparent for Aten-type asteroids. In figures 9 and 10, the semimajor axis and the eccentricity are fixed at 0.9 au (orbital period $\approx 0.85$ years) and $0.4 \mathrm{au}$, respectively, and the inclination is varied from $0^{\circ}$ to $60^{\circ}$. In figures 11 and 12 , the semimajor axis and the inclination are fixed at 0.8 au (orbital period $\approx 0.72$ years) and $10^{\circ}$, respectively, and the eccentricity is varied from 0.3 to 0.6 . In figures 13 and 14 , the eccentricity and the inclination are fixed at $0.6 \mathrm{au}$ and $20^{\circ}$, respectively, and the semimajor axis is varied from 0.65 au (orbital period $\approx 0.52$ years) to 0.95 au (orbital period $\approx 0.93$ years). In all cases examined, the orbital inclination, $i$, has a relatively small effect on the minimum $\Delta \mathrm{V}$ compared with eccentricity $e$ and semimajor axis $a$ (see figs. 7 and 11).

When only a two-body approximation is applied to the deflecting problem, the minimum $\Delta \mathrm{V}$ is linearly proportional to the miss distance, $\mathrm{R}_{\mathrm{m}}$ (ref. 6). For example, in the two-body approximation, deflecting a dangerous celestial body by $10 \mathrm{R}_{\oplus}$ requires exactly 10 times more $\Delta \mathrm{V}$ than that needed to deflect the object by $1 \mathrm{R}_{\oplus}$. When the gravitational effects of Earth are considered, the minimum $\Delta \mathrm{V}$ is linearly proportional not to the miss distance but to the impact parameter, $b_{\mathrm{i}}$. Figure 15 includes the minimum $\Delta \mathrm{V}$ (impact after perihelion) of a fictitious asteroid whose orbital elements are $a=1.5 \mathrm{au}, e=0.5$, and $i=20^{\circ}$ as the miss distance $\mathrm{R}_{\mathrm{m}}$ varies from $1 \mathrm{R}_{\oplus}$ to $100 \mathrm{R}_{\oplus}$. One Earth-Moon distance is approximately $60.27 \mathrm{R}_{\oplus}$. Analysis of equations (10) and (11) explains that the $\Delta \mathrm{V}$ requirement for a deflection of $\mathrm{NR}_{\oplus}$ is less than $\mathrm{N}$ times the $\Delta \mathrm{V}$ requirement for $1 \mathrm{R}_{\oplus}$ deflection. The reason is that $\mathrm{V}_{\text {esc }}$ is reduced as $\mathrm{R}_{\mathrm{m}}$ is increased; hence, $b_{\mathrm{i}}$ is not linearly proportional to $\mathrm{R}_{\mathrm{m}}$. For instance, at $\mathrm{t}=-1$ impulse time of the asteroid's orbital period, the $\Delta \mathrm{V}$ requirement for $1 \mathrm{R}_{\oplus}$ deflection is about $2.5187 \mathrm{~cm} / \mathrm{s}$ while the $\Delta \mathrm{V}$ requirements for $10 \mathrm{R}_{\oplus}$ and $100 \mathrm{R}_{\oplus}$ deflection are about $21.6146 \mathrm{~cm} / \mathrm{s}$ and $212.1871 \mathrm{~cm} / \mathrm{s}$, respectively.

\section{Short-Period Comets and Long-Period Comets}

Earth-crossing comets are classified into two types: short-period comets (SPCs), or those with an orbital period $<200$ years, and long-period comets (LPCs), or those defined here as having an orbital period $>200$ years. In figure 16, the histories of the minimum $\Delta \mathrm{V}$ (miss distance of Earth radius) for a fictitious SPC whose orbital elements are given by $a=4.0 \mathrm{au}, e=0.85$, and $i=0^{\circ}, 20^{\circ}, 40^{\circ}$, and $60^{\circ}$ are shown. For this example, the comets have an orbital period of approximately 8.0 years, perihelial distance of $0.6 \mathrm{au}$, and aphelial distance of $7.4 \mathrm{au}$. It is again noted that the $\Delta \mathrm{V}_{\mathrm{W}}$ component is small enough to be neglected. The effect of inclination on the SPC minimum $\Delta \mathrm{V}$ is also relatively small, as was observed previously for NEAs. Figure 17 includes the histories of optimal impulse angles corresponding to $\Delta \mathrm{V}$ in figure 16. As impulse time approaches impact time, the class of postperihelial impact has peaks in optimal impulse angle while the class of preperihelial impact has an optimal impulse angle more sensitive to the inclination. Like other ECOs, the SPC has a slightly larger magnitude of minimum impulse for the case of preperihelial impact than for the case of postperihelial impact. For the SPC, when the impulse time is very close to impact time, the case of preperihelial impact also has more variation in the optimal impulse angle than the case of postperihelial impact as the inclination varies. The data in figures 18 and 19 demonstrate that the same trends are apparent for LPCs, and the same trends may be expected for all 


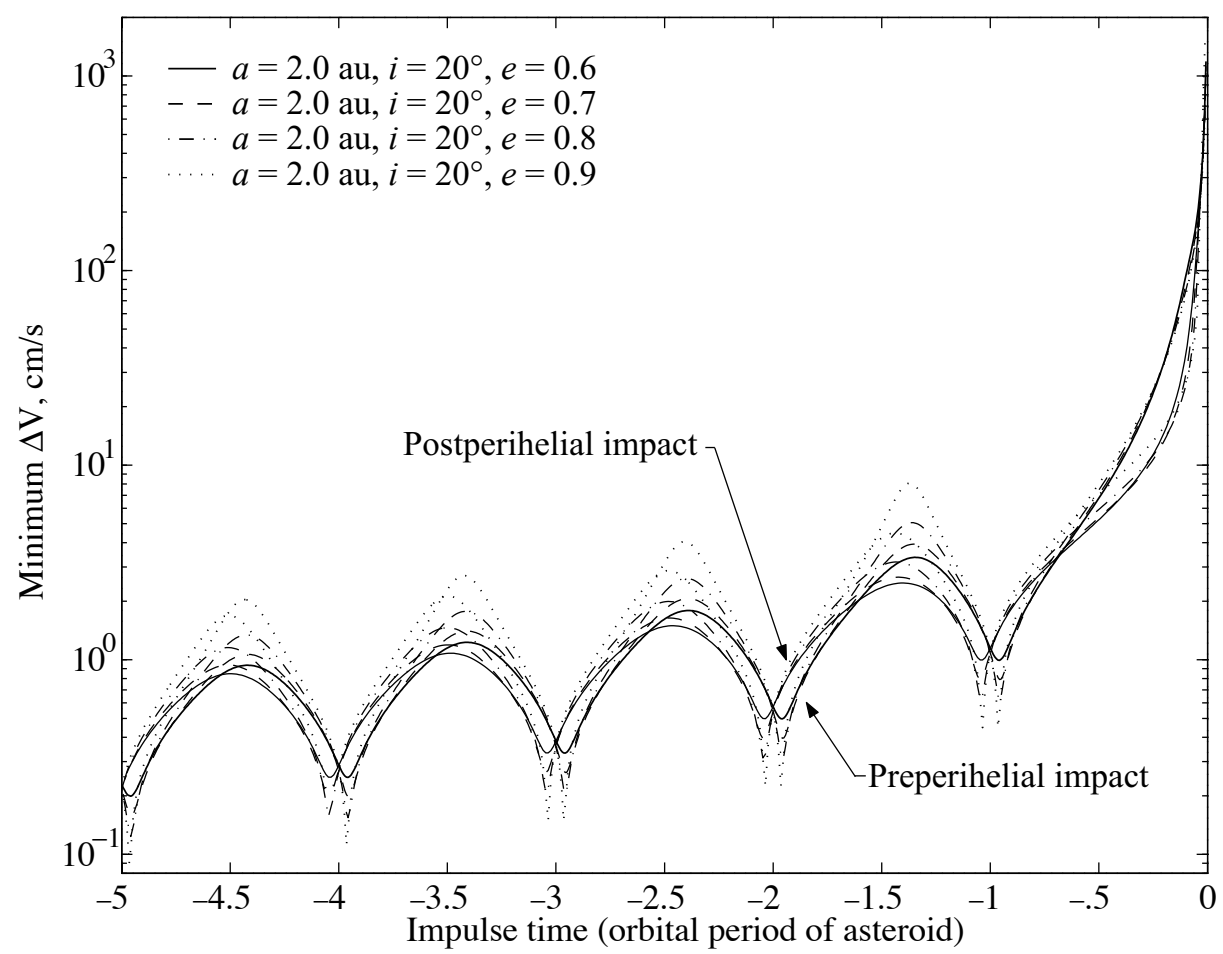

Figure 5. Minimum $\Delta \mathrm{V}$ : Apollo-type asteroids with $a=2.0$ au, $i=20^{\circ}, e=0.6,0.7,0.8,0.9$.

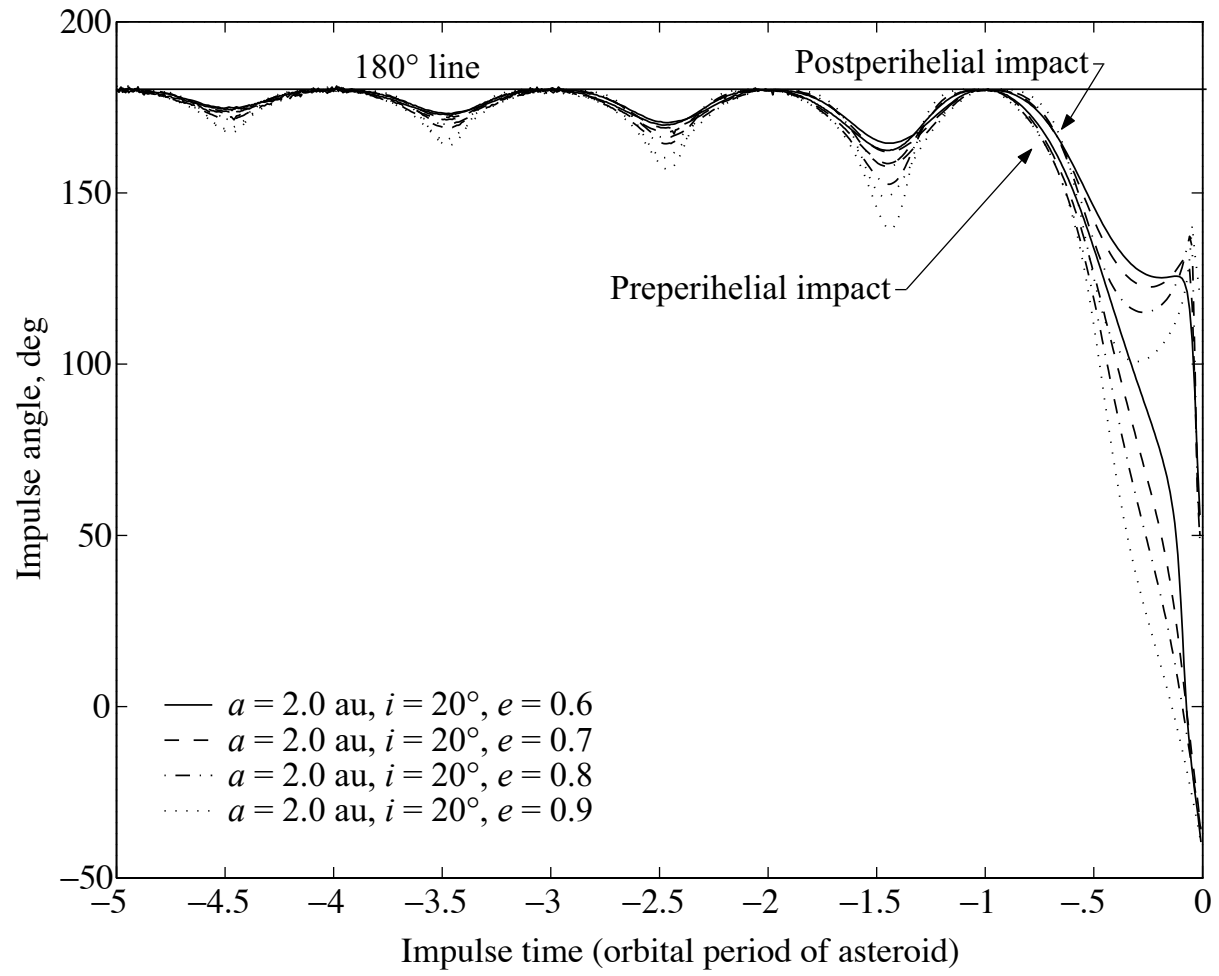

Figure 6. Impulse angle: Apollo-type asteroids with $a=2.0 \mathrm{au}, i=20^{\circ}, e=0.6,0.7,0.8,0.9$. 


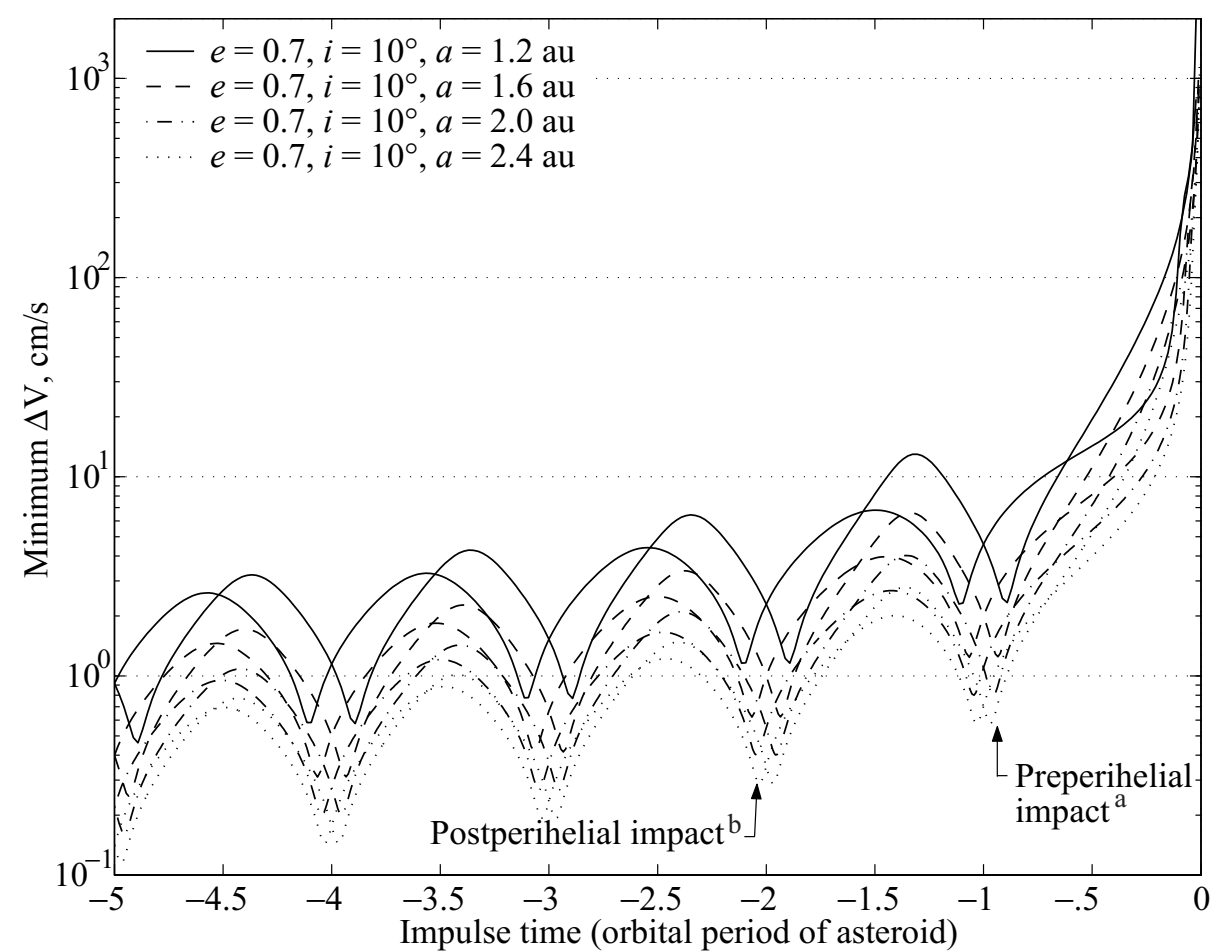

a Preperihelial impact: local minimum occurred just before a minus integer impulse time b Postperihelial impact: local minimum occurred just after a minus integer impulse time

Figure 7. Minimum $\Delta \mathrm{V}$ : Apollo-type asteroids with $e=0.7, i=10^{\circ}, a=1.2,1.6,2.0,2.4$ au.

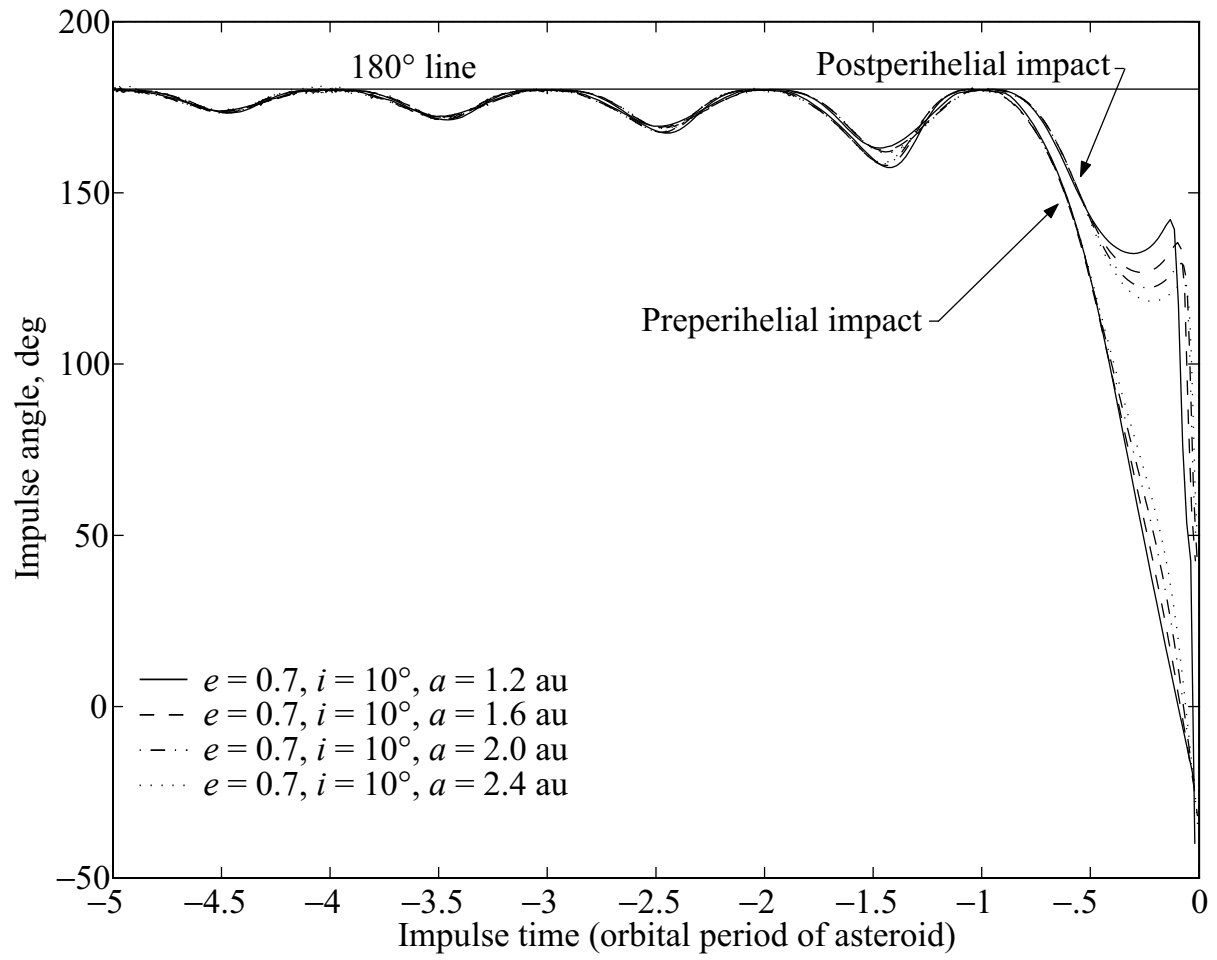

Figure 8. Impulse angle: Apollo-type asteroids with $e=0.7, i=10^{\circ}, a=1.2,1.6,2.0,2.4$ au. 


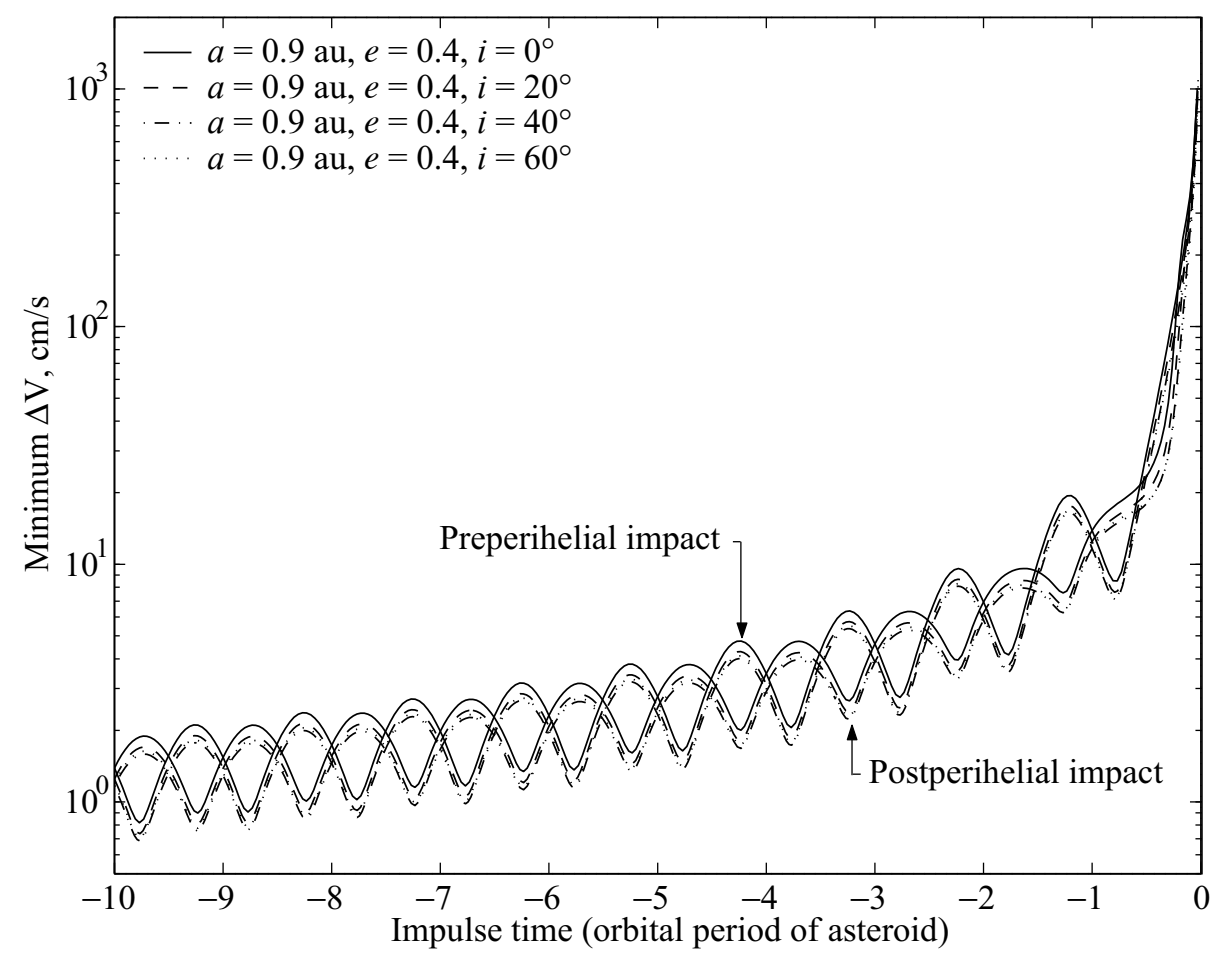

Figure 9. Minimum $\Delta \mathrm{V}$ : Aten-type asteroids with $a=0.9 \mathrm{au}, e=0.4, i=0^{\circ}, 20^{\circ}, 40^{\circ}, 60^{\circ}$.

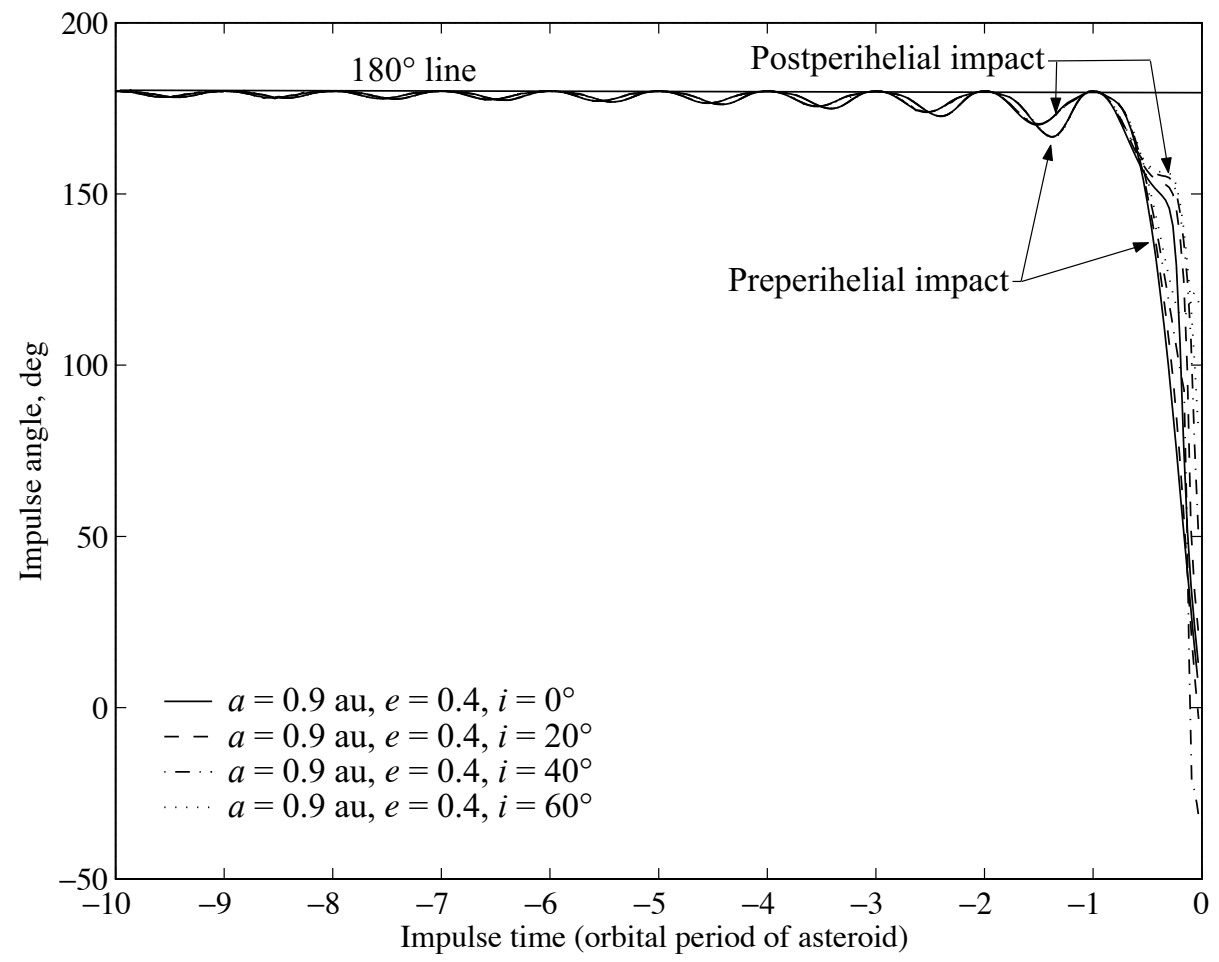

Figure 10. Impulse angle: Aten-type asteroids with $a=0.9$ au, $e=0.4, i=0^{\circ}, 20^{\circ}, 40^{\circ}, 60^{\circ}$. 


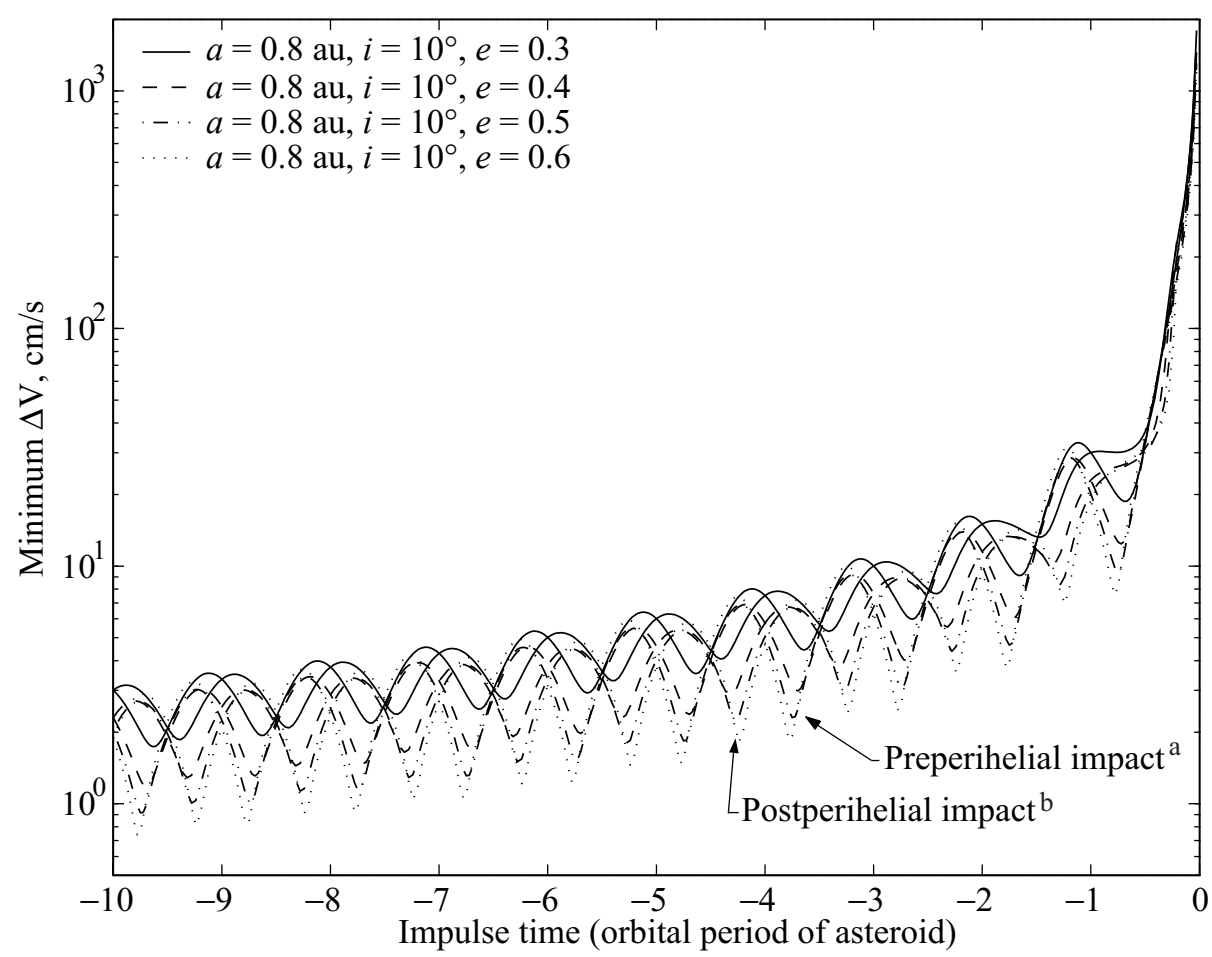

a Preperihelial impact: local minimum occurred just before a minus integer impulse time $\mathrm{b}$ Postperihelial impact: local minimum occurred just after a minus integer impulse time

Figure 11. Minimum $\Delta \mathrm{V}$ : Aten-type asteroids with $a=0.8$ au, $i=10^{\circ}, e=0.3,0.4,0.5,0.6$.

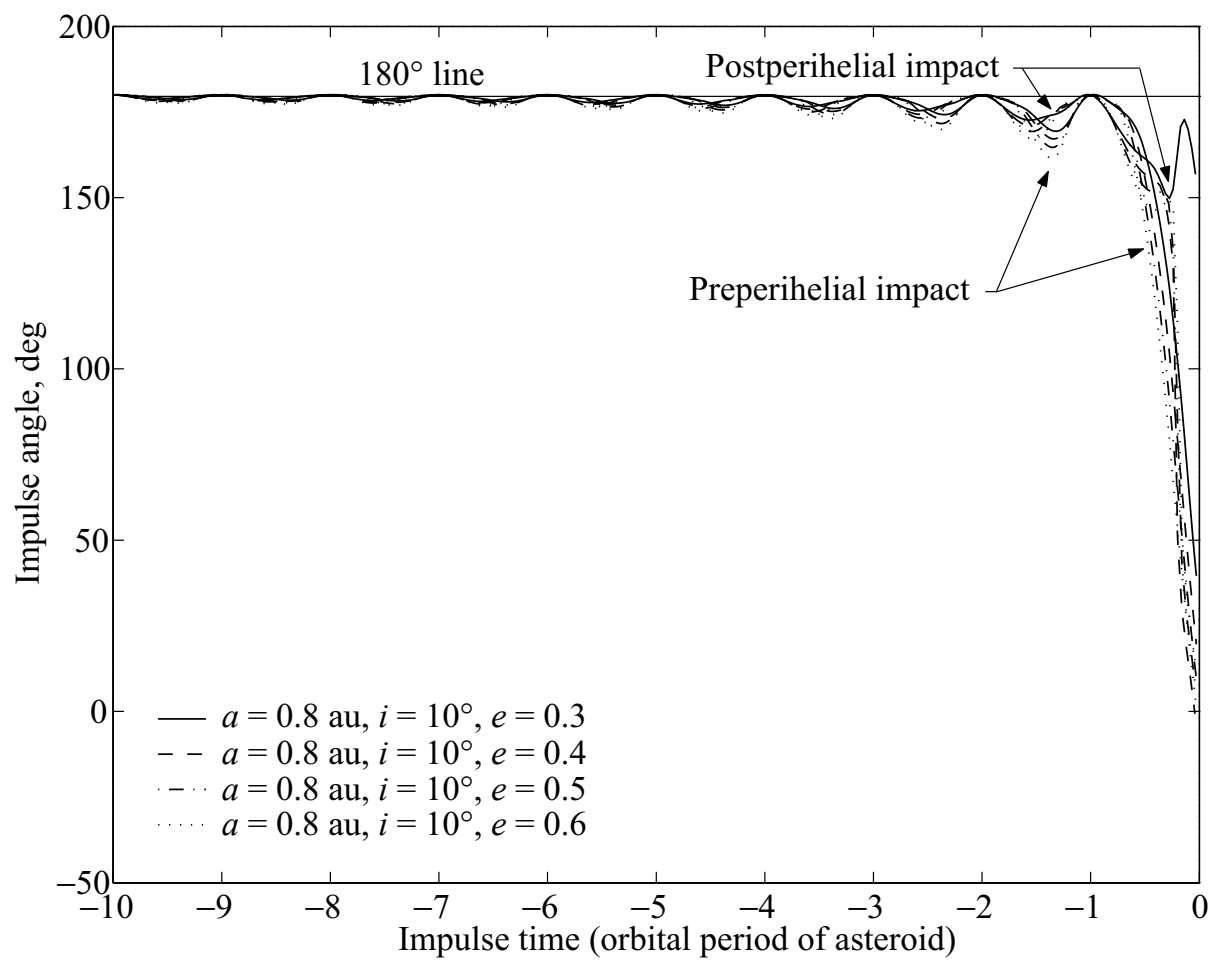

Figure 12. Impulse angle: Aten-type asteroids with $a=0.8$ au, $i=10^{\circ}, e=0.3,0.4,0.5,0.6$. 


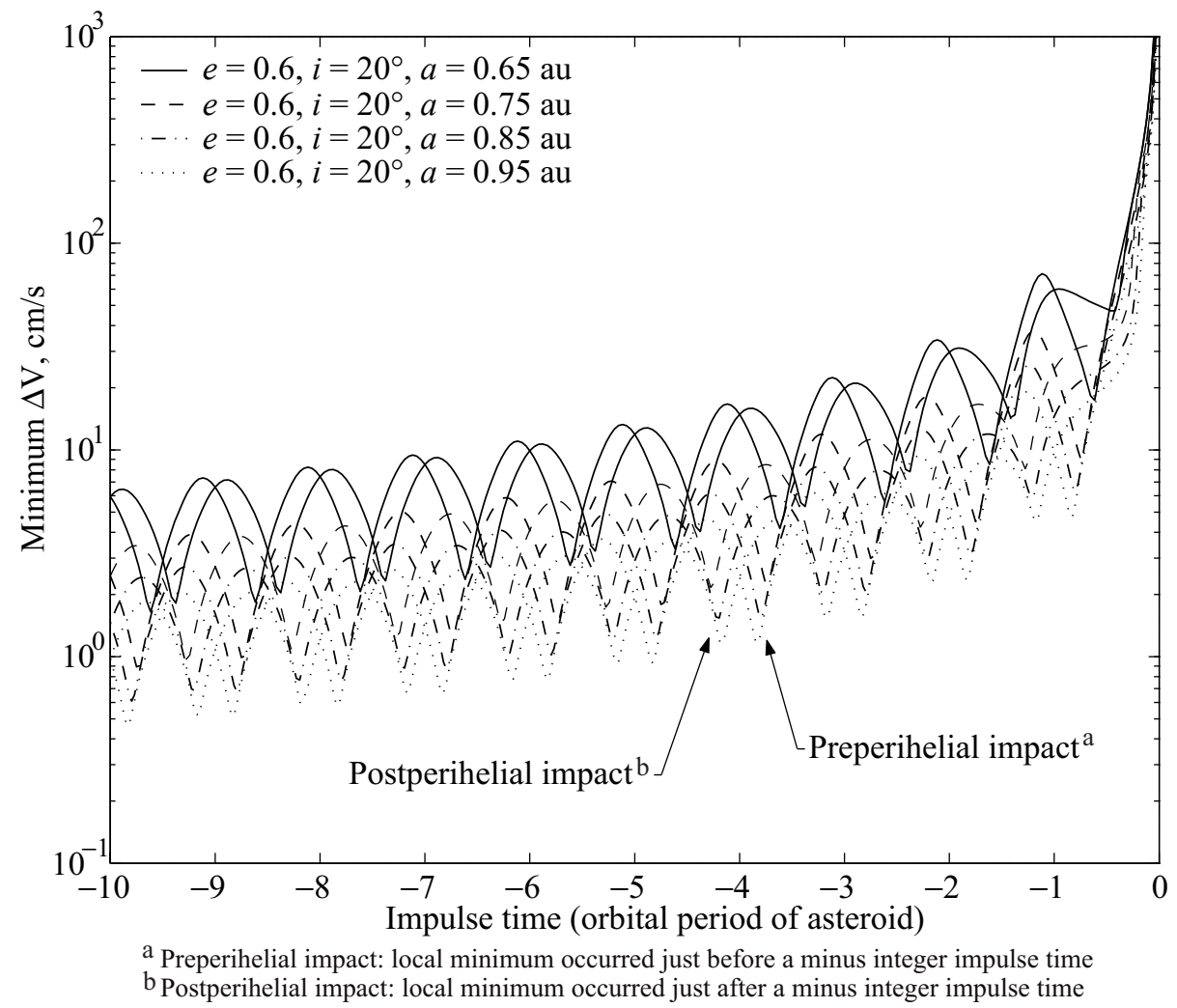

Figure 13. Minimum $\Delta \mathrm{V}$ : Aten-type asteroids with $e=0.6, i=20^{\circ}, a=0.65,0.75,0.85,0.95$ au.

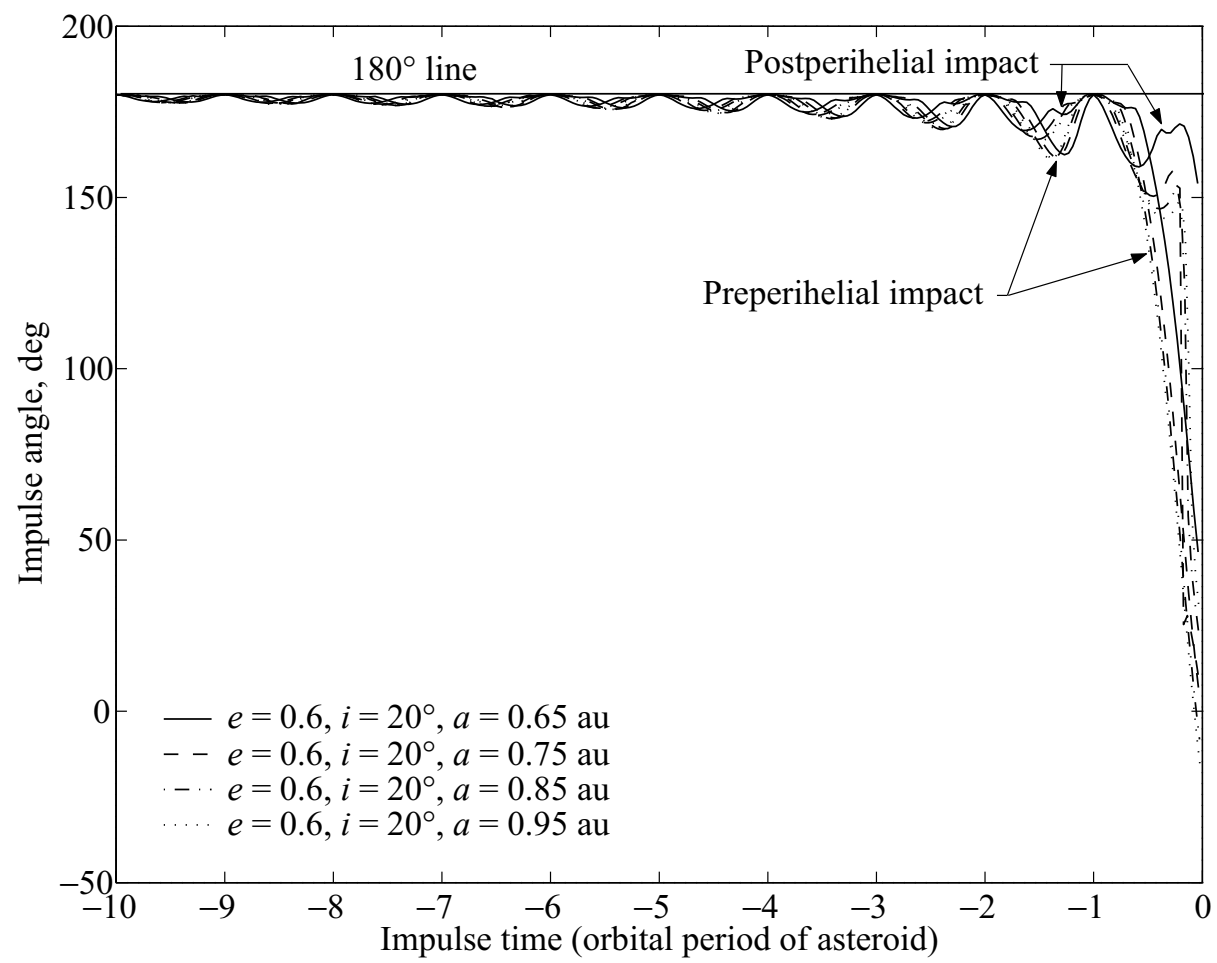

Figure 14. Impulse angle: Aten-type asteroids with $e=0.6, i=20^{\circ}, a=0.65,0.75,0.85,0.95 \mathrm{au}$. 


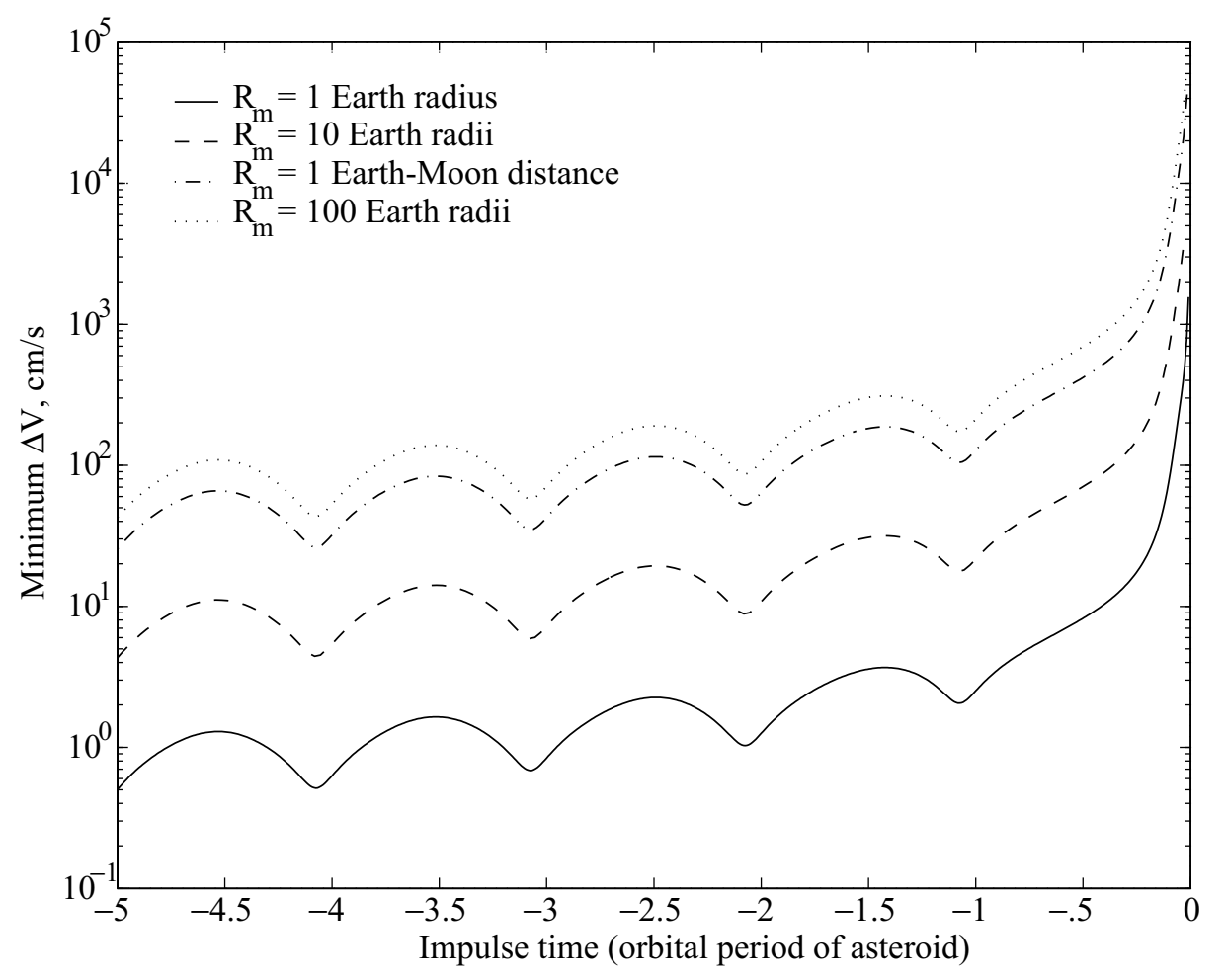

Figure 15. Minimum $\Delta \mathrm{V}$ : with respect to miss distance $\left(\mathrm{R}_{\mathrm{m}}\right)$.

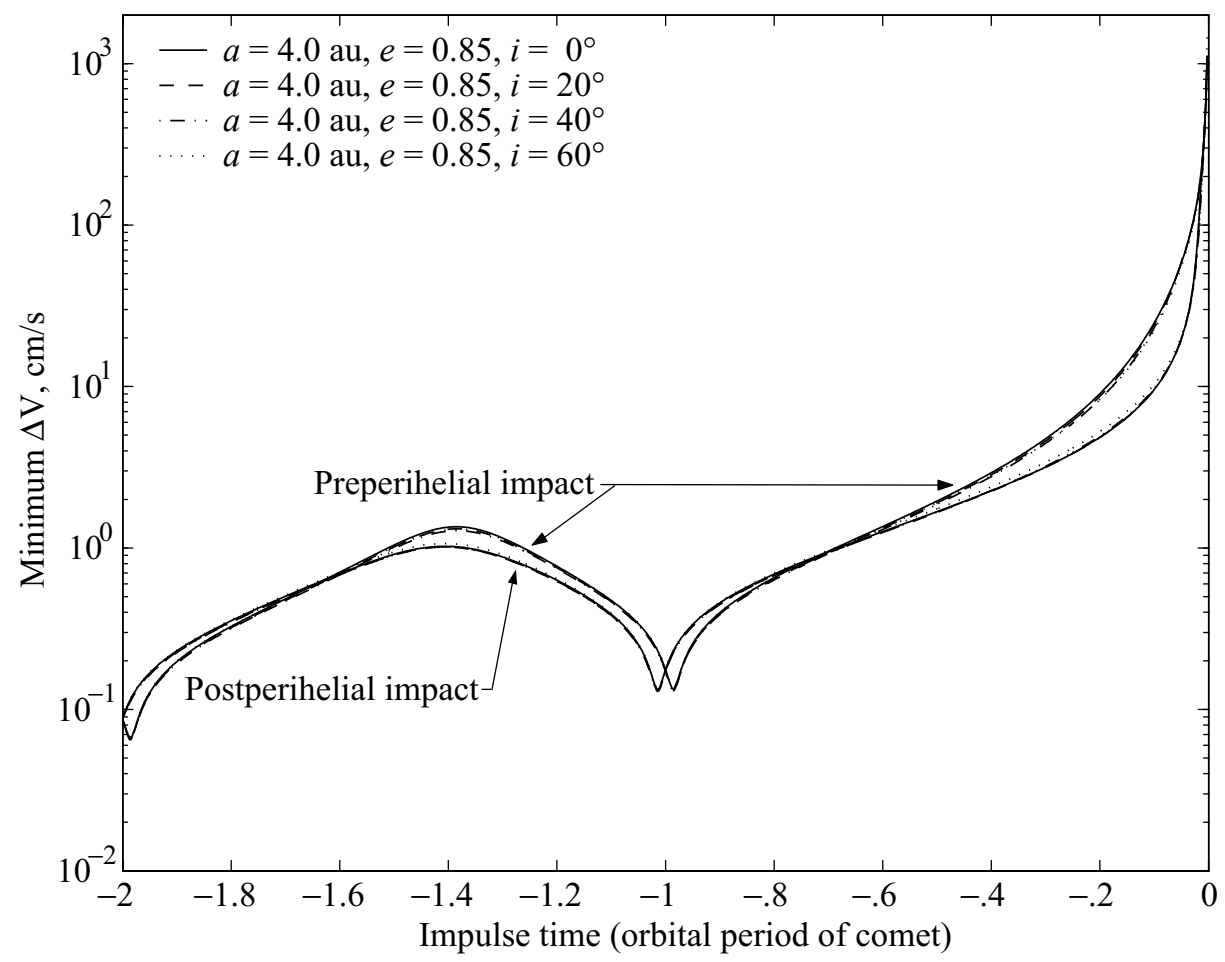

Figure 16. Minimum $\Delta \mathrm{V}$ : short-period comets with $a=4.0 \mathrm{au}, e=0.85, i=0^{\circ}, 20^{\circ}, 40^{\circ}, 60^{\circ}$. 


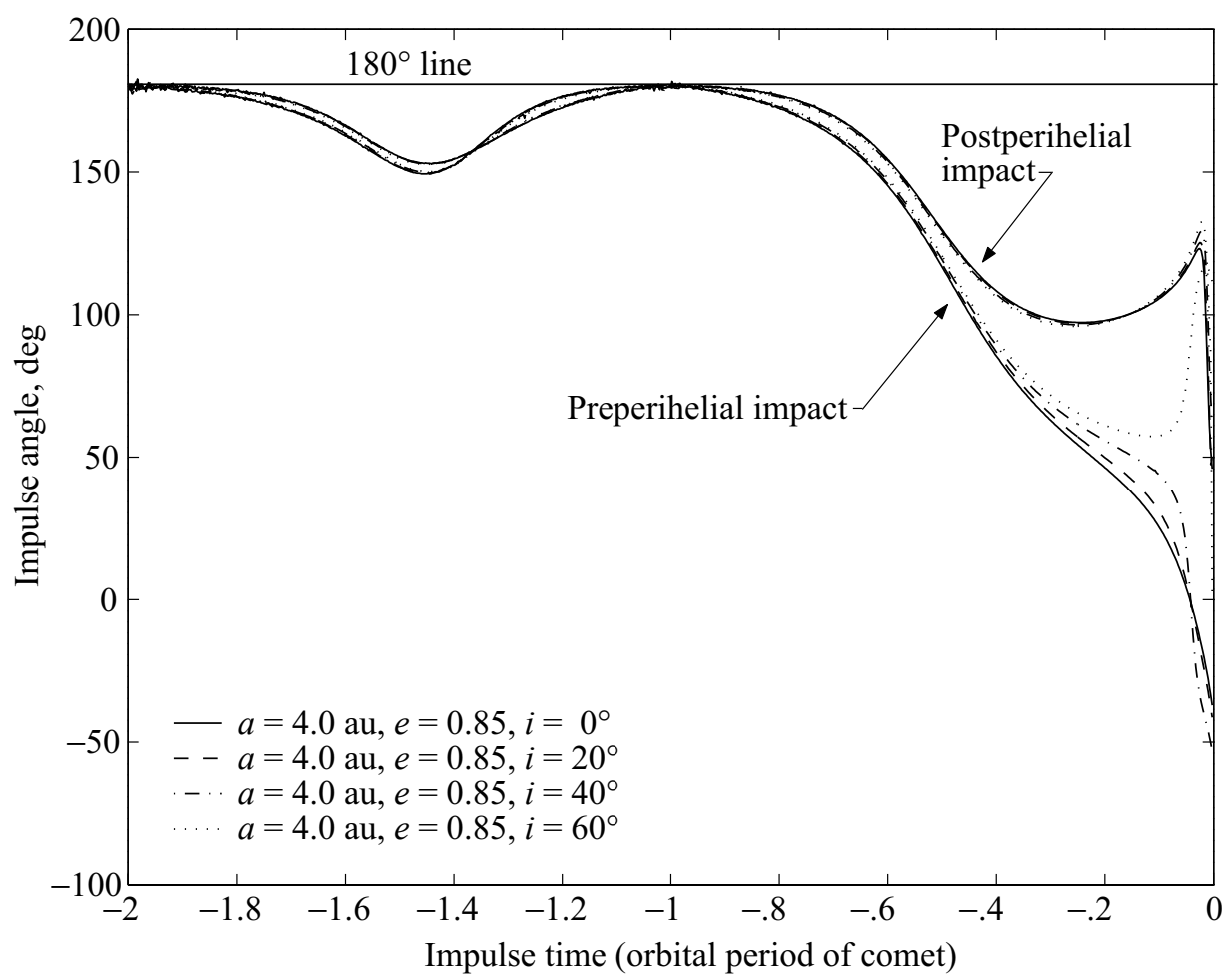

Figure 17. Impulse angle: short-period comets with $a=4.0 \mathrm{au}, e=0.85, i=0^{\circ}, 20^{\circ}, 40^{\circ}, 60^{\circ}$.

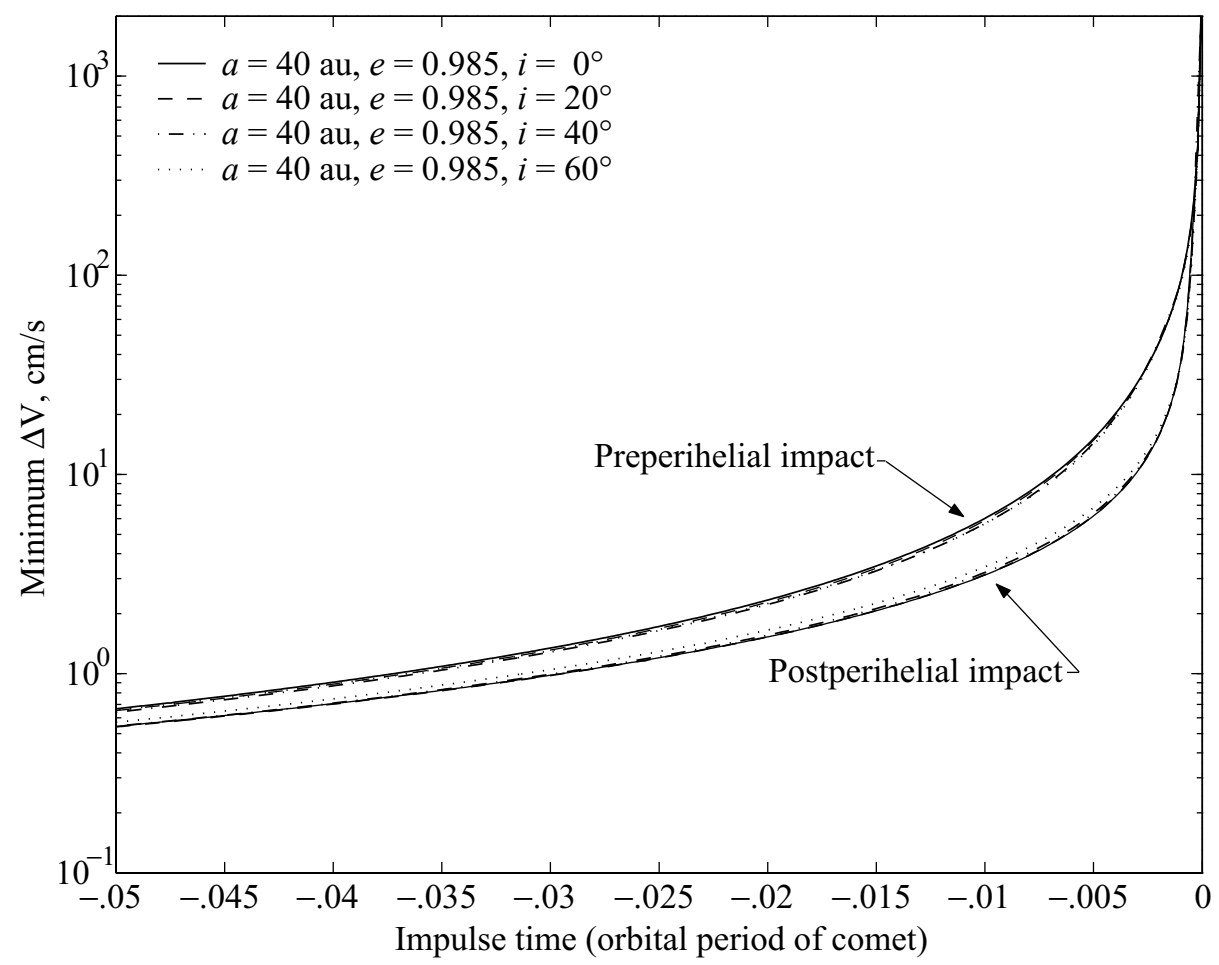

Figure 18. Minimum $\Delta \mathrm{V}$ : long-period comets with $a=40$ au, $e=0.985, i=0^{\circ}, 20^{\circ}, 40^{\circ}, 60^{\circ}$. 


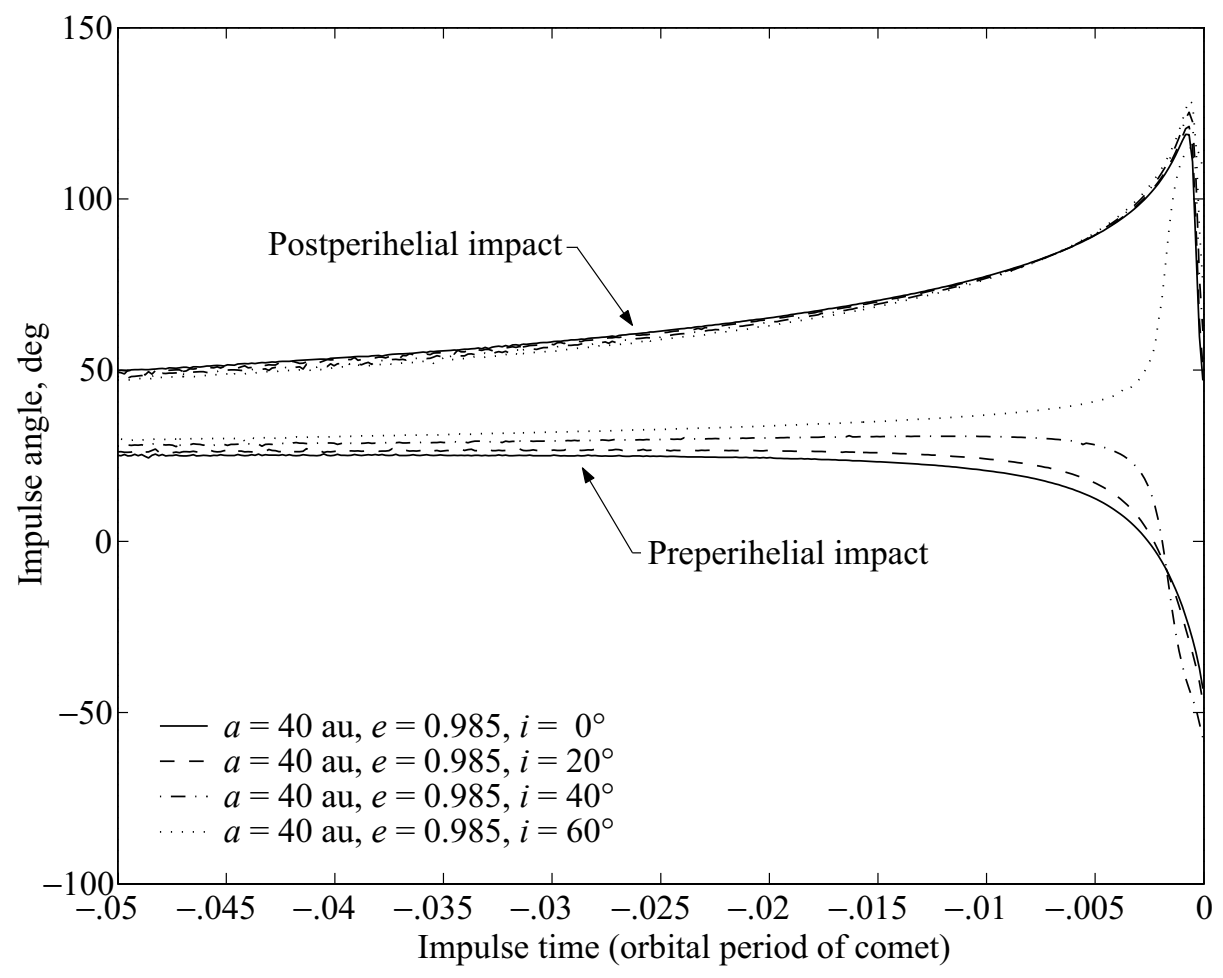

Figure 19. Impulse angle: long-period comets with $a=40$ au, $e=0.985, i=0^{\circ}, 20^{\circ}, 40^{\circ}, 60^{\circ}$.

LPCs. In the figures, the semimajor axis and the eccentricity are fixed at 40 au (orbital period $\approx 253$ years, perihelial distance $\approx 0.6 \mathrm{au}$, aphelial distance $\approx 79.4 \mathrm{au}$ ) and 0.985 , respectively, and the inclination is varied from $0^{\circ}$ to $60^{\circ}$.

For ECOs, the data in figures 20 and 21 show $\Delta \mathrm{V}$ and impulse angle histories of less than a 10 -year impulse time. The $\Delta \mathrm{Vs}$ for the fictitious LPC $(0.05$ orbital period $\approx 12.7$ years $)$ are monotonically decreasing functions of impulse time, while the $\Delta \mathrm{Vs}$ for the fictitious NEA have the cyclic component varying with respect to orbital period. For a given miss distance and impulse time, the slower ECOs with respect to the Earth usually require a larger $\Delta \mathrm{V}$. The order of magnitude of $\Delta \mathrm{V}$ is not significantly different for either the asteroids or the comets considered here. For an impulse time less than 10 years, the impulse angle of the LPC is mostly monotonically decreasing with respect to impulse time while the angle of the NEA has fluctuations due to its short orbital period. At the final stage, where impulse time approaches impact time, asteroids and comets have no significant differences in optimal $\Delta \mathrm{V}$ and impulse angles. Additionally, the $\Delta \mathrm{V}$ required for deflecting Earth-approaching asteroids and comets is dramatically increased, and impulse angle is quickly decreased.

\section{Interceptor Strategies}

It is important to estimate the required interceptor mass or energy to deflect or disrupt asteroids/ comets on a collision course with Earth. With the estimated mass or energy, we can investigate the appropriate strategies for the deflection missions. The interceptor parameter of $\mathrm{M}_{\mathrm{ht}}, \mathrm{E}_{\mathrm{laser}}, \mathrm{M}_{\mathrm{k}}, \mathrm{M}_{\mathrm{nso}}$, and $\mathrm{M}_{\mathrm{nss}}$ required for a given ECO mass and $\Delta \mathrm{V}$ are expressed for high-thrust engine, laser ablation, 


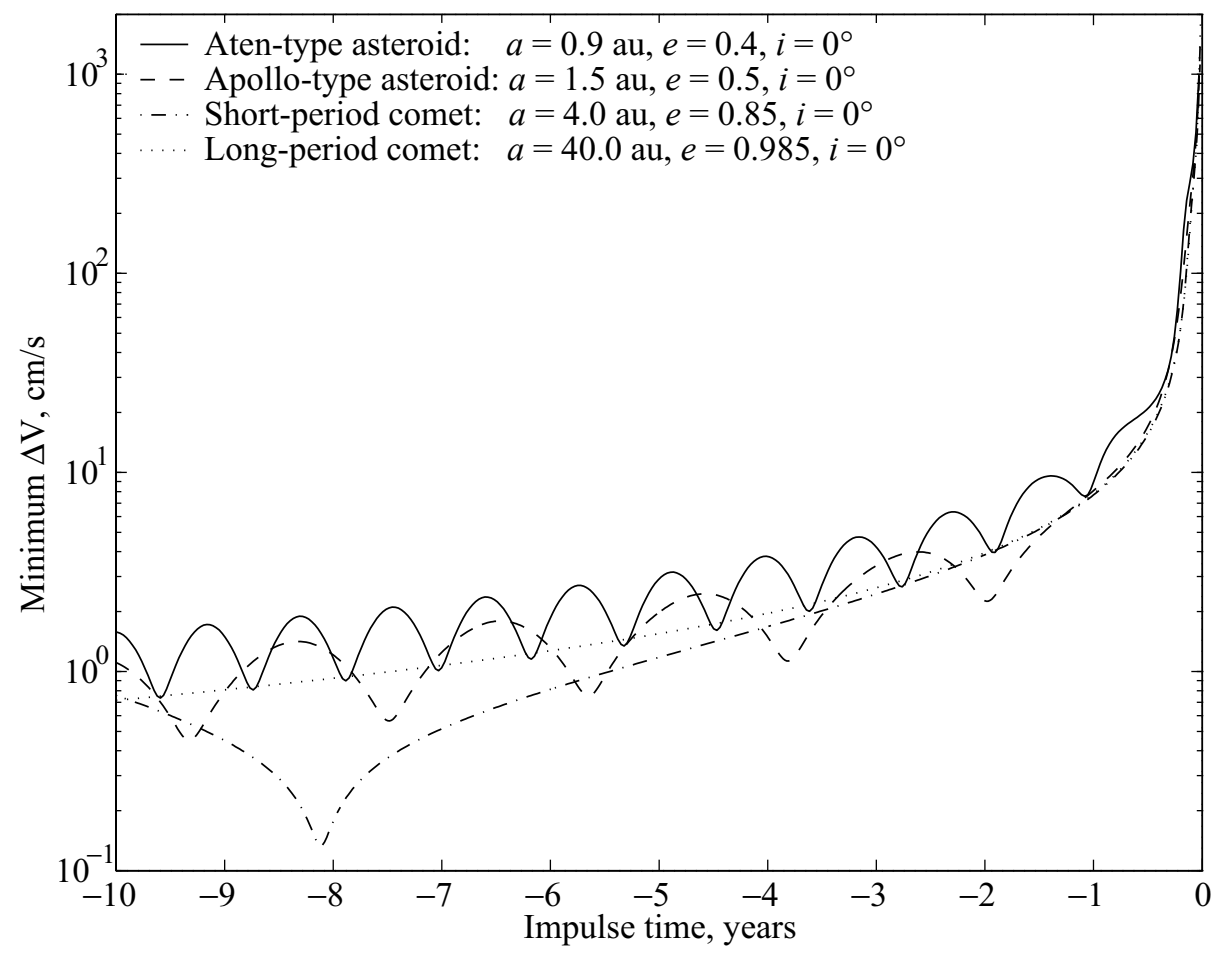

Figure 20. For comparison: minimum $\Delta \mathrm{V}$ of postperihelial impact for various ECOs.

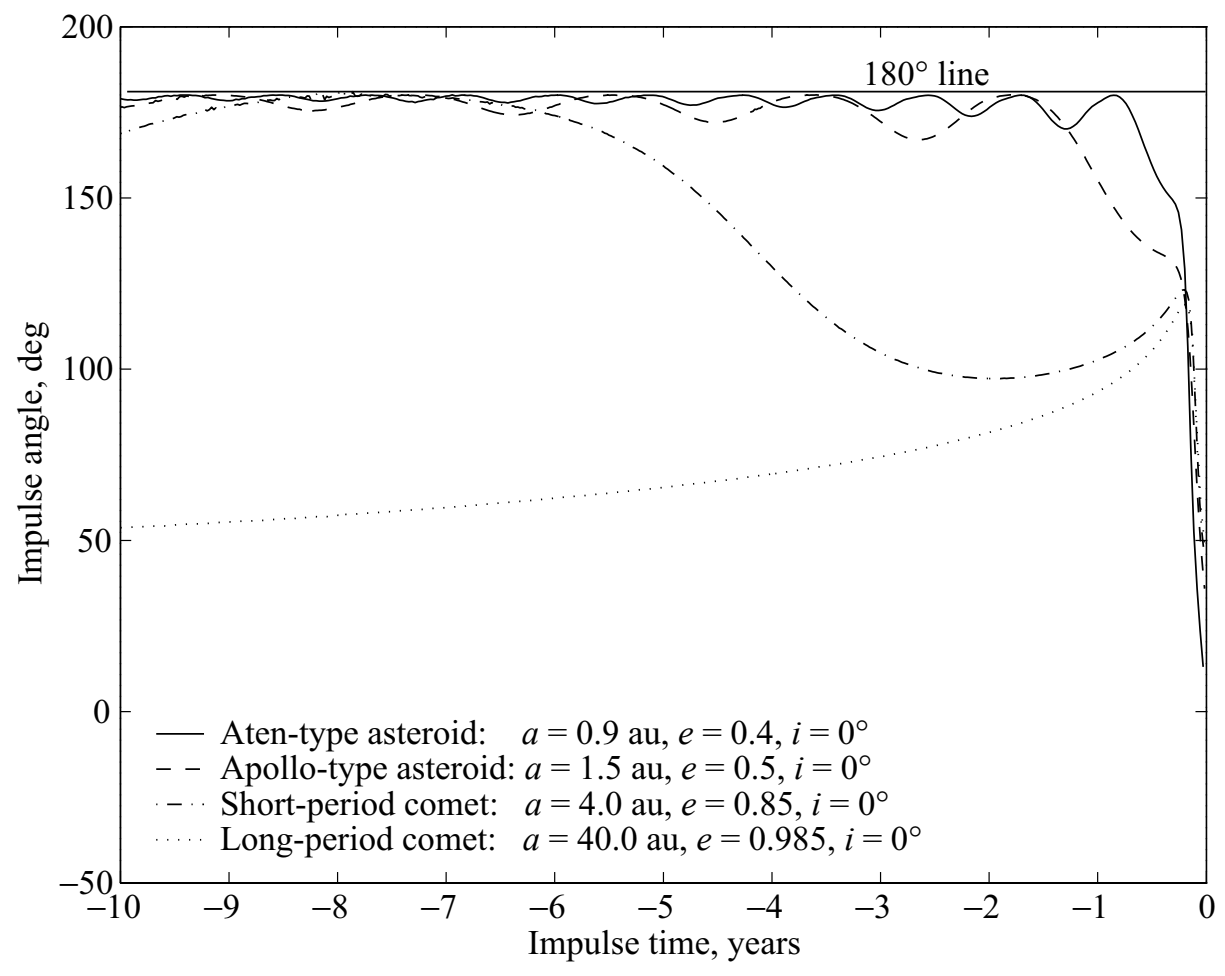

Figure 21. For comparison: impulse angle of postperihelial impact for various ECOs. 
kinetic-energy deflection, standoff nuclear detonation, and slightly subsurface nuclear burst (refs. 3, 16, and 17) as follows:

$$
\begin{gathered}
\mathrm{M}_{\mathrm{ht}} \approx \frac{m \Delta \mathrm{V}}{\mathrm{c}_{\mathrm{e}}}\{\mathrm{kg}\} \\
\mathrm{E}_{\text {laser }} \approx \frac{m \Delta \mathrm{V}}{\mathrm{c}_{\mathrm{m}}}\{\mathrm{J}\} \\
\mathrm{M}_{\mathrm{k}} \approx \frac{m}{\varepsilon}\left(\frac{\mathrm{v}_{\mathrm{e}} \Delta \mathrm{V}}{\mathrm{v}^{2}}\right)\{\mathrm{kg}\} \\
\mathrm{M}_{\mathrm{nso}} \approx \frac{\mathrm{c}_{\mathrm{p}}}{\varepsilon \phi} m \Delta \mathrm{V}\{\mathrm{kg}\} \\
\mathrm{M}_{\mathrm{nss}} \approx \frac{m}{2 \varepsilon} \frac{\mathrm{v}_{\mathrm{e}} \Delta \mathrm{V}}{\phi}\{\mathrm{kg}\}
\end{gathered}
$$

The interceptor parameter indicates mass $(\mathrm{kg})$ of fuel for a high-thrust engine, energy $(\mathrm{J})$ for a laser ablation technique, spacecraft total mass $(\mathrm{kg})$ for a kinetic-energy deflection, mass $(\mathrm{kg})$ of nuclear explosive for a standoff nuclear detonation, and a subsurface nuclear detonation. In these equations, other parameter values are defined or assumed as follows (ref. 17). The velocity of the ejecta is assumed as $\mathrm{v}_{\mathrm{e}} \approx 100 \mathrm{~m} / \mathrm{s}$ for subsurface bursts, $m$ is the mass of the asteroid/comet, $m=\frac{4}{3} \pi \rho\left(\frac{\mathrm{D}}{2}\right)^{3}$, density is assumed as $3 \times 10^{3} \mathrm{~kg} / \mathrm{m}^{3}$ for stony asteroids ( $200 \mathrm{~kg} / \mathrm{m}^{3}$ for comets), $\mathrm{D}$ is diameter of the asteroid/comet, about a fraction $\varepsilon \approx 0.5$ of the total energy could go into the kinetic energy of ejected material, nuclear explosions provide a specific energy $\phi \approx 8 \times 10^{12} \mathrm{~J} / \mathrm{kg}$, a compression wave velocity in the asteroid/comet material is $\mathrm{c}_{\mathrm{p}} \approx 2 \mathrm{~km} / \mathrm{s}, \mathrm{c}_{\mathrm{e}}$ is the exhaust velocity of a high-thrust engine, $\mathrm{c}_{\mathrm{m}}$ is the laser momentum coupling coefficient (assumed in ref. 16 as $\mathrm{c}_{\mathrm{m}}=5 \mathrm{dyn}-\mathrm{s} / \mathrm{J}$ ), $\mathrm{v}$ is the orbital velocity of an asteroid/comet, and $\Delta \mathrm{V}$ is the orbital velocity increment to deflect an asteroid/comet on a collision course with Earth by 1 Earth radius. It is assumed that the velocity of the interceptor is much less than that of celestial objects and can be neglected.

The optimization method mentioned previously yields the $\Delta \mathrm{V}$ to be used in equations (15) through (19). Note that equations (15) through (19) implicitly have estimation errors to give the final interceptor parameter, and the impulse is assumed to be applied with the optimal impulse angle discussed previously. Two fictitious asteroids (Aten-type asteroid: $a=0.9 \mathrm{au}, e=0.4, i=0^{\circ}$, orbital period $=0.85$ years; Apollo-type asteroid: $a=1.5 \mathrm{au}, e=0.5, i=0^{\circ}$, orbital period $=1.84$ years) and two comets (SPC: $a=4.0 \mathrm{au}, e=0.85, i=0^{\circ}$, orbital period $=8.0$ years, LPC: $a=40 \mathrm{au}, e=0.985, i=0^{\circ}$, orbital period $=253$ years $)$ are used. With $\Delta \mathrm{V}$ calculated with respect to impulse time, equations (15) through (19) can be applied to estimate the final interceptor parameter to deflect the asteroid/comet by at least 1 Earth radius. Inclined asteroid/comet orbits $\left(i \neq 0^{\circ}\right)$ should give approximately the same interceptor parameter as that of a planar orbit because there are no significant differences in $\Delta \mathrm{V}$ histories for both the inclined and planar orbits.

Figures 22 and 23 include the histories of the final interceptor parameter in order to deflect $1-\mathrm{km}$ diameter stony asteroids (Aten-type or Apollo-type) by 1 Earth radius, while figures 24 and 25 include 


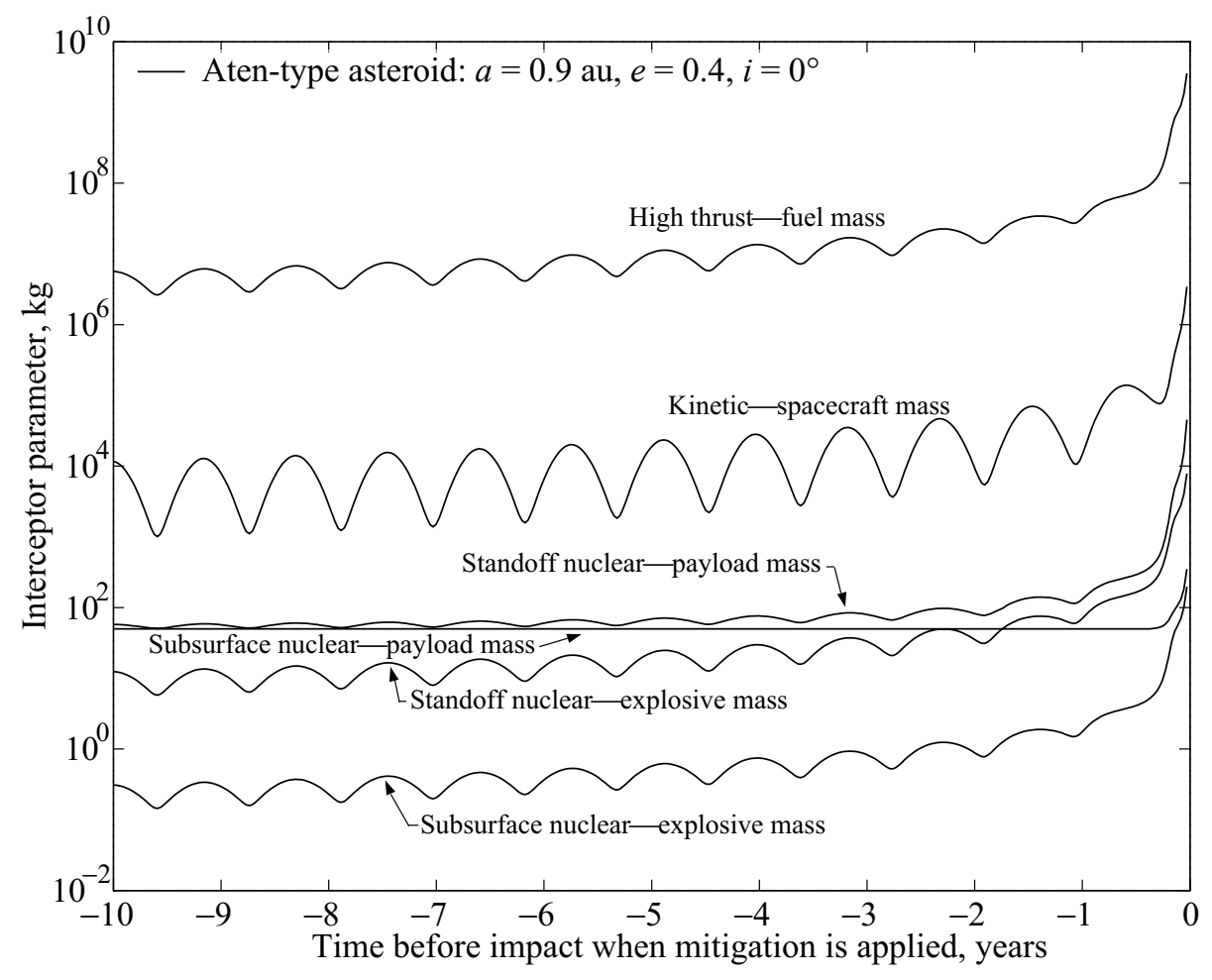

Figure 22. Various interceptor masses for 1-km stony Aten-type asteroid.

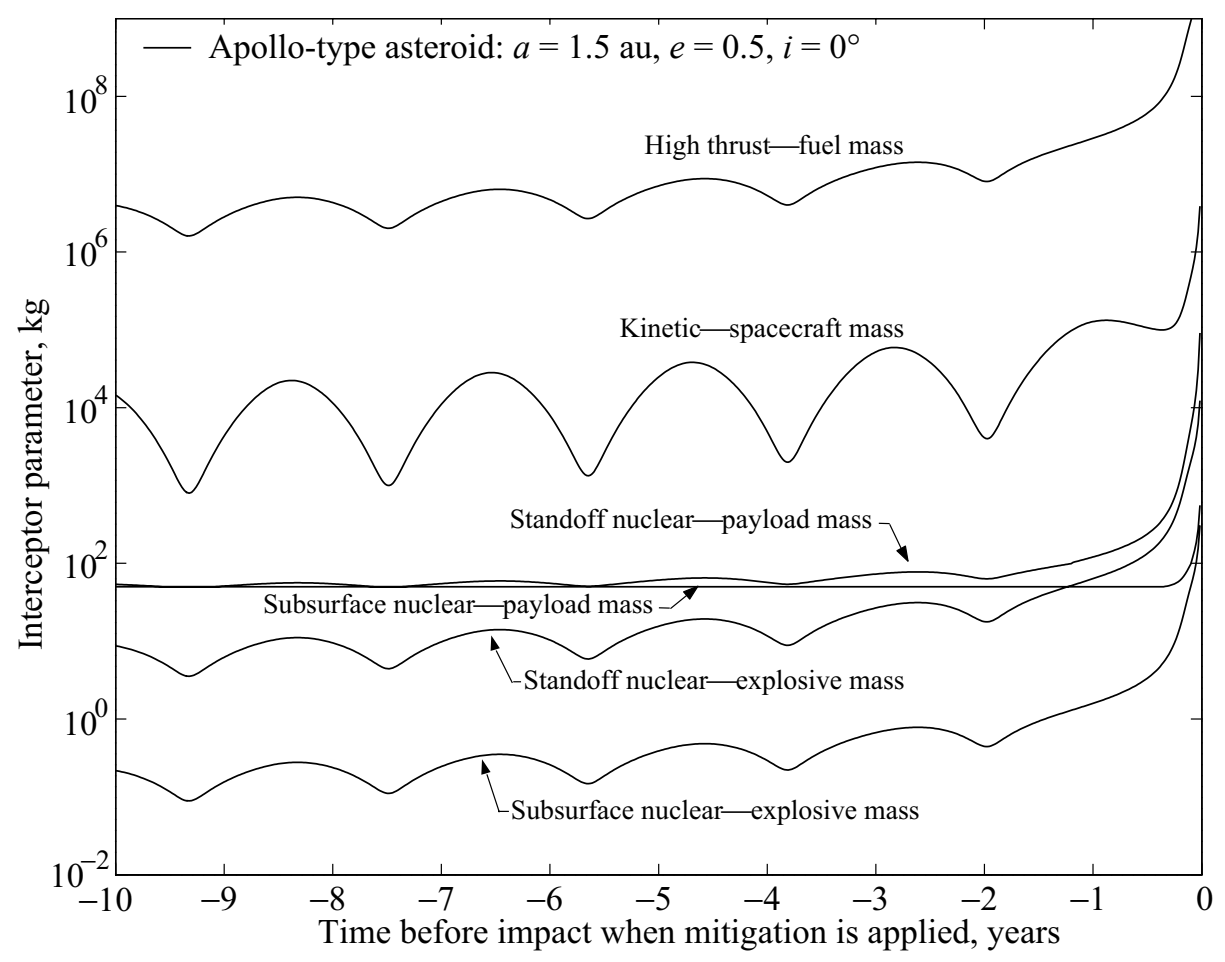

Figure 23. Various interceptor masses for 1-km stony Apollo-type asteroid. 


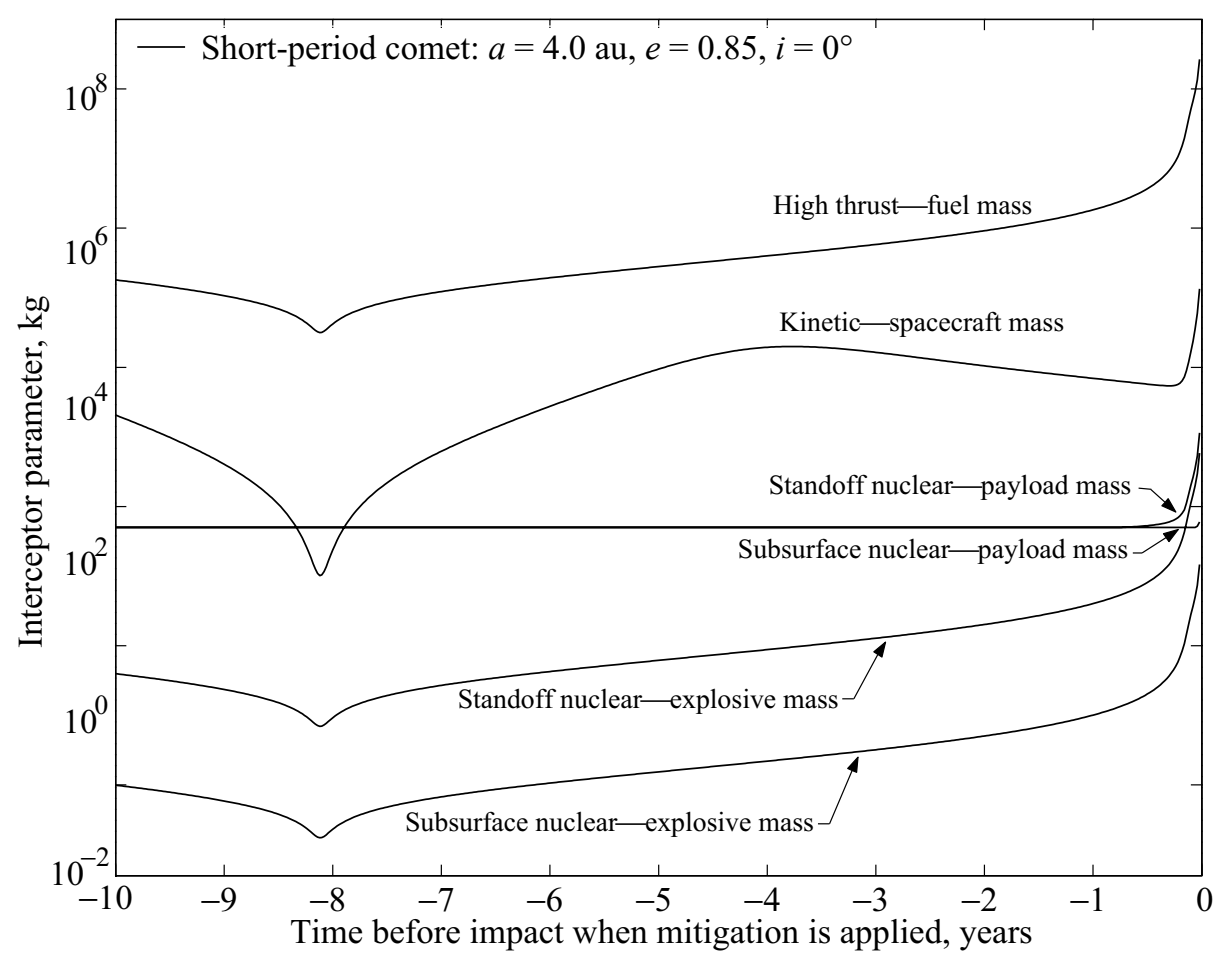

Figure 24. Various interceptor masses for 1-km short-period comet.

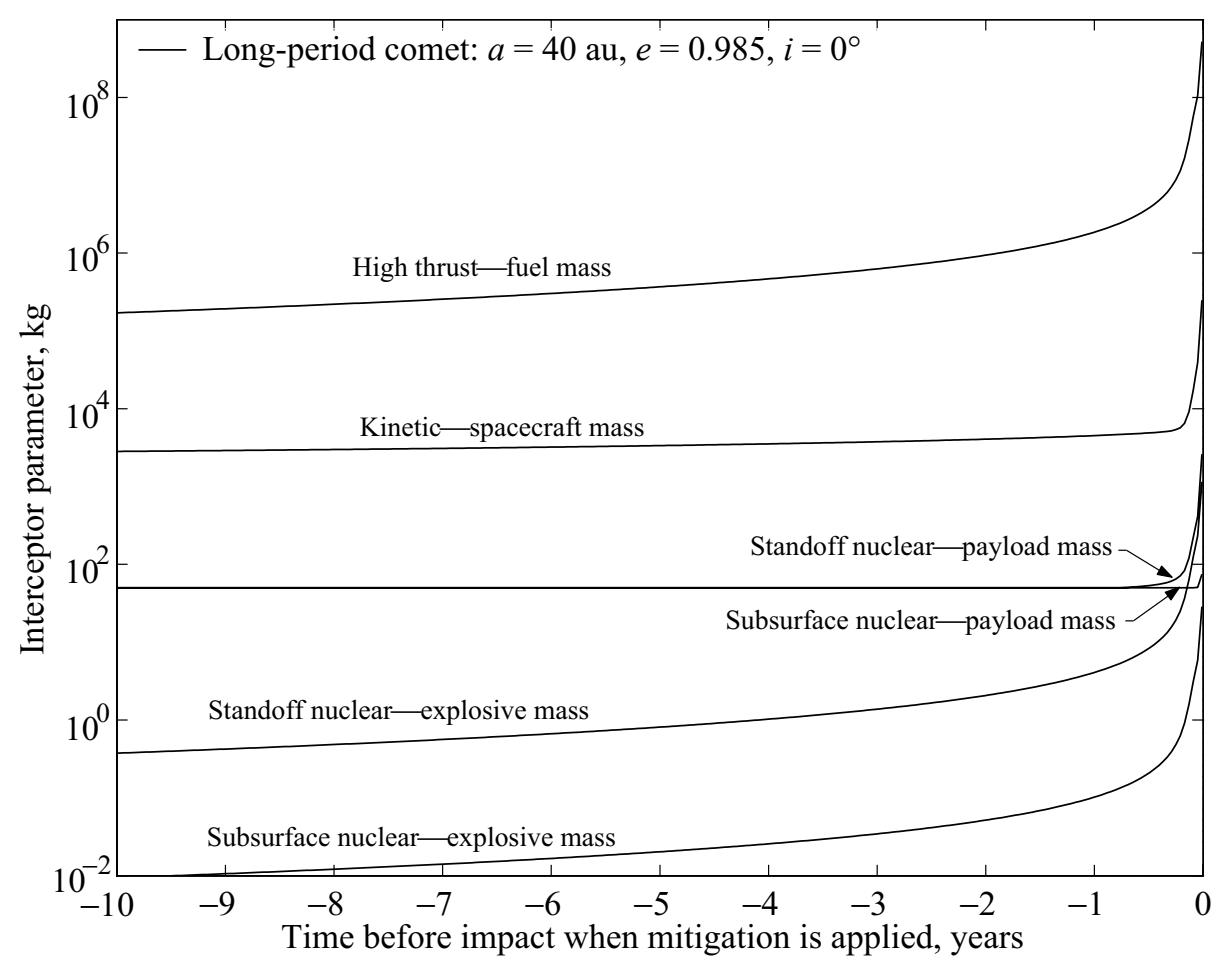

Figure 25. Various interceptor masses for 1-km long-period comet. 
similar histories for 1-km diameter comets (an SPC and an LPC). The final intercepter parameter for Apollo-type asteroids shows the same pattern as those for Aten-type asteroids, with less fluctuations with respect to impulse time. For these figures, the payload masses of nuclear equipment are estimated in table 1, which aligns the data to show the relationship between nuclear yield and nuclear device weight (ref. 18). Note that a minimum payload mass of $50 \mathrm{~kg}$ is assumed when nuclear yield is less than $10 \mathrm{kt}$. The final interceptor parameters also vary according to the fluctuations of minimum $\Delta \mathrm{V}$ required for the space deflection missions. For both the asteroid and comet, a high-thrust method requires much more mass than other methods identified (kinetic-energy deflection, standoff nuclear detonation, and subsurface nuclear burst) because of the fuel expenses for a soft landing and smaller exhaust velocity (ref. 3). Kinetic-energy deflection methods require hundreds of times less mass than high-thrust methods for asteroid deflection, and several orders of magnitude less mass for comet deflection. Nuclear detonation methods reduce the final payload mass by several orders of magnitude compared with the kinetic-energy deflection method. The explosive mass of a slightly subsurface nuclear burst is less than that for standoff nuclear detonation by several factors of 10 for both asteroid and comet deflection. However, the payload mass of both nuclear methods could be $50 \mathrm{~kg}$ when impulse time is greater than 1 year before impact. An interesting phenomenon is that, for the SPC case, spacecraft mass for kinetic-energy deflection could be less than nuclear payload mass (fig. 24) when deflection is provided at the $\Delta V$ 's local minimum. The reason for this phenomenon is that the velocity of the comet is relatively high at perihelion, and final mass of kinetic deflection is inversely proportional to the square of orbital velocity. Generally, the interceptor masses for comet deflection are less than those for asteroid deflection, because densities of comets are assumed to be much less than those of asteroids. Because the mass of an asteroid and comet is linearly proportional to the cube of its diameter, it is easy to estimate the final interceptor masses for different sizes of asteroids and comets. For example, $0.1-\mathrm{km}$ stony asteroids require one thousand times less final mass than 1-km stony asteroids. For asteroid missions, the "optimal time" for application of the $\Delta \mathrm{V}$ is the earliest possible perihelion for impulse times greater than 1 orbital period, while for LPC missions, the optimal time is simply the earliest possible time. Surprisingly, for an LPC mission, the interceptor mass for the kinetic-energy deflection is approximately the same when the impulse time goes from 1 to 10 years before collision with Earth, although $\Delta \mathrm{V}$ is increased during the period (see fig. 18). The reason for this phenomenon is that the orbital velocity of the comet is increased accordingly as it travels toward its perihelion, and the interceptor mass of kinetic-energy deflection is inversely proportional to the square of orbital velocity.

Table 1. Approximate Nuclear Yield Versus Nuclear Device Weight ${ }^{\mathrm{a}}$

\begin{tabular}{|c|c|}
\hline Nuclear yield, kt & Payload weight, $\mathrm{kg}$ \\
\hline 10 & 50 \\
100 & 100 \\
1000 & 1000 \\
10000 & 20000 \\
\hline
\end{tabular}

${ }^{\mathrm{a}}$ From reference 18.

\section{Use of a Hybrid Propellant Module}

A hybrid propellant module (HPM) is a future reusable in-space transportation concept being studied under NASA's Revolutionary Aerospace Systems Concepts (RASC) Program. The HPM can store indefinitely both chemical and electrical propellants and provide propulsion with attached modular orbital transfer/engine stages. The HPM can utilize a chemical propellant (liquid hydrogen/liquid oxygen) for 
fast orbital transfers and can use an electrical propellant (low thrust) for prepositioning or to return the HPM for reuse and refueling. A chemical transfer module (CTM) serves as a high energy injection stage when attached to the HPM, while a solar electric propulsion (SEP) module serves as a low-thrust transfer stage when attached to the HPM. Although propulsive $\Delta \mathrm{V}$ is much less efficient for deflecting asteroids and comets, the cost of utilizing an already available infrastructure might be favorable compared with developing a separate defensive capability. A preliminary investigation of how an HPM-based transportation system could be utilized is provided. It is assumed that the dry mass of an HPM is $4000 \mathrm{~kg}$, the dry mass of a CTM is $4400 \mathrm{~kg}$, the chemical propellant mass of a CTM is $30000 \mathrm{~kg}$, and the effective exhaust velocity, $\mathrm{c}_{\mathrm{e}}$, is $4.4 \mathrm{~km} / \mathrm{s}$ for the CTM. An HPM/CTM/SEP vehicle stack can travel to an ECO by using the low-thrust, high-specific impulse provided by the SEP and the high thrust provided by the CTM and HPM chemical propellant. The idea of having abundant propellant sources in deep space may offset the disadvantages of a chemical propellant when combined with electric propulsion.

After rendezvous with the ECO, the combined vehicle could use its CTM as a high-thrust engine with a full $30000 \mathrm{~kg}$ load of chemical propellant to change the orbit of the asteroid/comet. According to figures 22 through 25 , by using a CTM as a high-thrust engine, it can be estimated that $\approx 0.13$-km asteroid $(\approx 0.3-\mathrm{km}$ comet) defense mission could be performed 2 years before collision. The same CTM could be used for $\approx 0.06-\mathrm{km}$ stony asteroid $(\approx 0.15-\mathrm{km}$ comet $)$ deflection just 6 months before collision. An asteroid of about a $1-\mathrm{km}(0.2-\mathrm{km})$ diameter can be deflected by direct spacecraft (HPM with CTM, total dry mass is about $8400 \mathrm{~kg}$ ) impact on the target for longer than a 2-year (6-month) impulse time if the impulse is applied to an asteroid at its perihelion. An approximate $1-\mathrm{km}$ comet can be deflected by kinetic energy using HPM with CTM for longer than 6 months of impulse time. These cases assume that all the fuel of a combined HPM with CTM can be consumed to travel longer or faster to dangerous objects. With an impulse time of longer than 2 years, HPM (dry mass of $4000 \mathrm{~kg}$ ) itself could perturb orbits of asteroids/ comets of $0.3 \mathrm{~km}$ in size using direct kinetic impact on the objects. By assuming that the HPM/CTM stack could deliver a 20000-kg nuclear warhead, this in-space transportation system could be used for a $1-\mathrm{km}$ asteroid or a $2-\mathrm{km}$ comet deflection mission for any short impulse time.

An HPM-based in-space transportation system could be available in 10 to 20 years. For comparative purposes, it is useful to determine what deflection strategies are currently possible. Payload mass is directly related to propellant mass, so the final mass of an interceptor can be converted into a rough estimate of interceptor cost. An interceptor payload would require 3 times more mass into deep space and 10 times more mass into low-Earth orbit (ref. 19). The Russian Energia launch vehicle was the most powerful launch vehicle and had a payload capacity of 80000 to $90000 \mathrm{~kg}$ for low-Earth orbits (ref. 12). With this launch capability, about $9000 \mathrm{~kg}$ of maximum final interceptor mass can be reasonably assumed. The data in figures 22 through 25 indicate that a $0.1-\mathrm{km}$ asteroid or $0.3-\mathrm{km}$ comet defense mission could be performed for any short impulse time by using kinetic-energy technology. A 1-km asteroid would require at least 2 years of impulse time if the $\Delta \mathrm{V}$ is applied at the asteroid's perihelion, while a $1-\mathrm{km}$ comet would require at least 1 year. These results coincide with previous studies stating that kinetic-energy impacts are suitable to deflect rocky asteroids of up to about $0.1 \mathrm{~km}$ in size (ref. 5). It was also shown that today's technology is sufficient to defend asteroids of less than $0.1 \mathrm{~km}$ in diameter for warning times on the order of several years (ref. 12). A 1-km asteroid or a 2-km comet defense mission could be prepared for a few months impulse time by using nuclear detonations. Nuclear explosives offer energies great enough for much larger asteroids/comets and shorter impulse times. The studies in this paper may be slightly different from previous studies due to different approximations and assumptions. Generally, for any warning time, we can roughly say that the kinetic-energy deflection would be preferable for smaller ECOs (less than a few hundred meters in size); the nuclear strategy would be required for large objects (greater than $1 \mathrm{~km}$ in size) or for very short impulse times. 


\section{Use of a Laser Ablation Technique}

Although nuclear detonations and kinetic-energy impacts appear to be the most practical near-term impact protection options, both methods have uncertainties in the momentum change imparted and can potentially fragment the asteroid/comet. This fragmentation could create multiple impacts, which ultimately could be more hazardous than the original object. Additionally, it is envisioned that comets and asteroids could provide a virtually limitless supply of resources for the future exploration and colonization of space. An orbit modification technique that could also facilitate the utilization of nonthreatening comets and asteroids would likely function in a controlled manner. One approach to alter the trajectory of the object in a highly controlled manner is to use pulsed laser ablative propulsion. This technique has been studied to remove space debris from low-Earth orbit (ref. 20). A sufficiently intense laser pulse ablates the surface of the space debris by causing plasma blowoff. The momentum change from a single laser pulse is very small. However, the cumulative effect is very effective because the laser can yield 10 to $1000 \mathrm{pulses} / \mathrm{s}$ over several minutes (ref. 21). The dynamic reaction from multiple laser hits reduces the perigee altitude of the orbiting debris. Once perigee is reduced sufficiently $(\approx 200 \mathrm{~km})$, atmospheric drag deorbits the debris. The highest pulse energy currently available is about $20 \mathrm{~kJ} /$ pulse operated at under $0.02 \mathrm{~Hz}$ (ref. 20). In the future, a much more advanced version of this same technique may be used for the deflection/orbit modification of asteroids/comets on a collision trajectory with Earth. The laser ablation technique could overcome the mass penalties associated with other nondisruptive approaches because no propellant is required to generate the $\Delta \mathrm{V}$ (the material of the celestial object is the propellant source). Additionally, laser ablation is effective against a wide range of surface materials and does not require any landing or physical attachment to the object.

For deflection of distant asteroids and comets, the power and optics requirements of a laser ablation system on the ground or near Earth may be too extreme to contemplate in the next decades. One hybrid solution would be to permit a spacecraft to carry a laser as a payload to a particular celestial body. The spacecraft would require an advanced propulsion system capable of rapid rendezvous with the asteroid/ comet and an extremely powerful energy generation system. The spacecraft would orbit or station-keep with the object at a "small" standoff distance. In this section, it is assumed that a spacecraft with a laser ablation tool has already rendezvoused with the asteroid/comet. Figure 26 includes the required laser energy for various ECOs to deflect them by 1 Earth radius; it is demonstrated that the required laser energy also varies according to the required $\Delta \mathrm{V}$. The size of the celestial bodies in figure 26 is assumed to be $1 \mathrm{~km}$. Again, it is easy to estimate the final interceptor energy for different sizes of ECO because the energy of an ECO is linearly proportional to the cubic of its diameter. Figure 27 houses an explanatory detail of figure 26 for an Apollo-type asteroid and an LPC, using fixed impulse times, and describes the required energy for laser ablation to deflect the given size of an asteroid and comet by 1 Earth radius. Figure 28 includes the achieved energy for a given laser power and operation period. From figures 27 and 28, we can easily estimate the power of laser for a selected case. For instance, if we can use a 1-year impulse time, about $2.5 \times 10^{3}$ GJ of energy (fig. 27) is required for a $0.1-\mathrm{km}$ stony asteroid to be deflected by 1 Earth radius. The data in figure 28 can be used to determine what laser power is required and how long the laser should be continuously operated in order to achieve $2.5 \times 10^{3} \mathrm{GJ}$ of energy. If we choose a laser system with $1 \mathrm{MW}$ of power $(100 \mathrm{~kJ} /$ pulse and $10 \mathrm{~Hz}$ laser repetition frequency), it would take about 30 days of operation to provide $2.5 \times 10^{3} \mathrm{GJ}$ of energy. If we choose a laser system with $10 \mathrm{MW}$ of power, it would take about 3 days of operation to provide $2.5 \times 10^{3} \mathrm{GJ}$ of energy. If a laser system with average power of $1 \mathrm{MW}$ could be continuously used for asteroid deflection 2 years before collision with Earth, it would take about 8 days to provide a $0.1-\mathrm{km}$ stony Apollo-type asteroid with enough energy to avoid the collision. If the same equipment is available 6 months before the collision, it would take 60 days for the same asteroid. For deflection of a 1-km LPC, a 25-MW laser system needs to be operated 


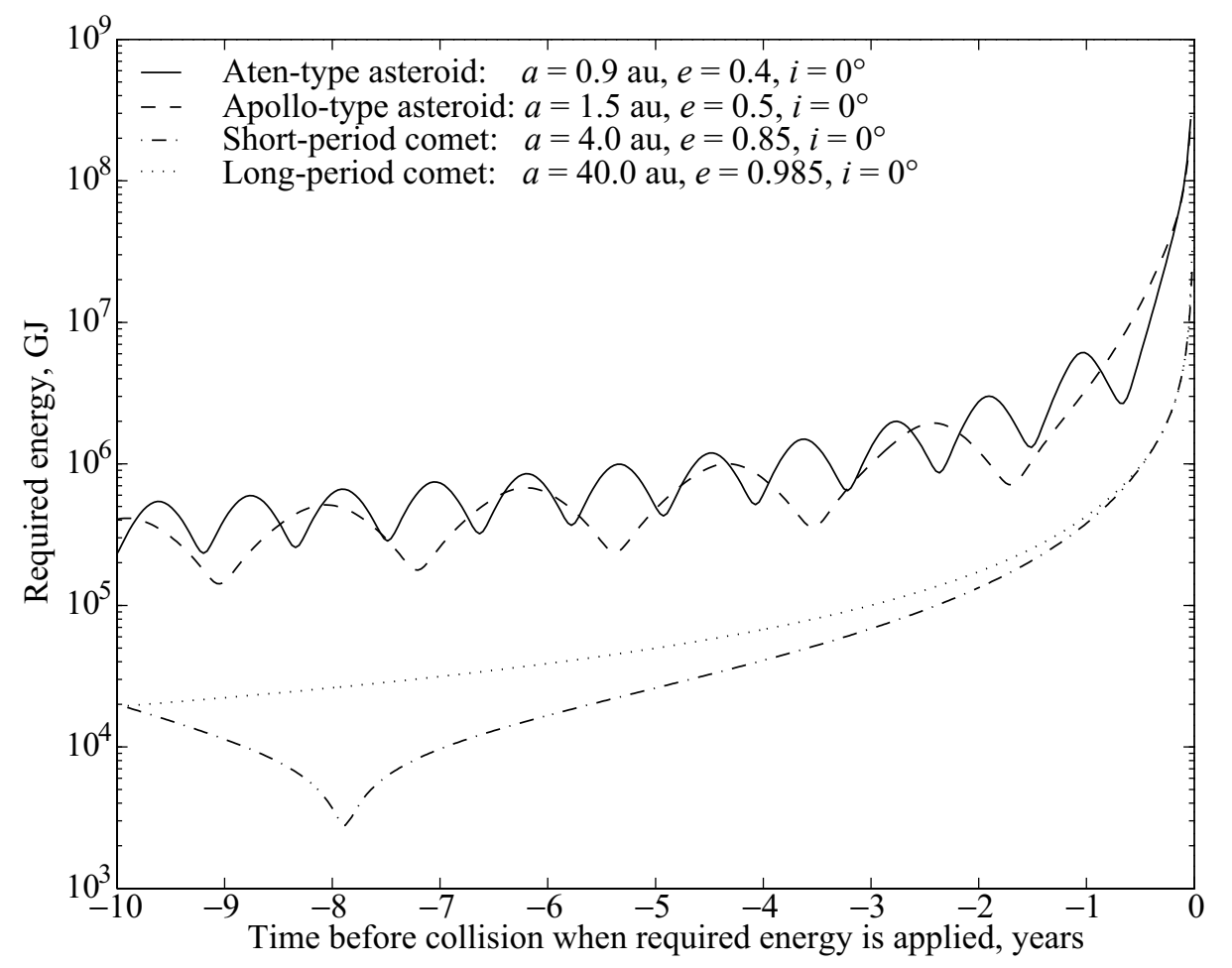

Figure 26. Required laser energy of postperihelial impact for 1-km ECOs.

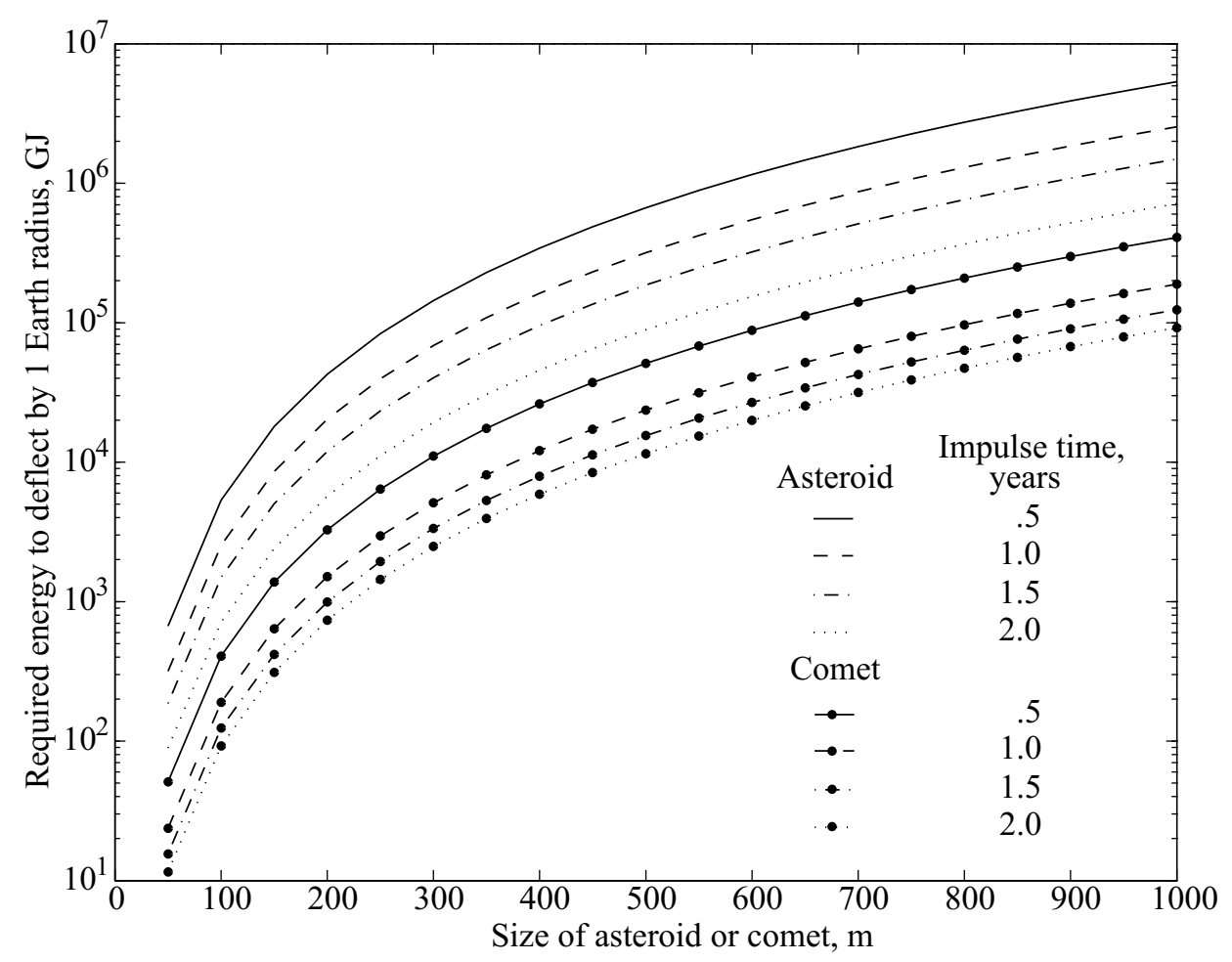

Figure 27. Required laser ablation energy versus size for Apollo-type asteroid and long-period comet. 


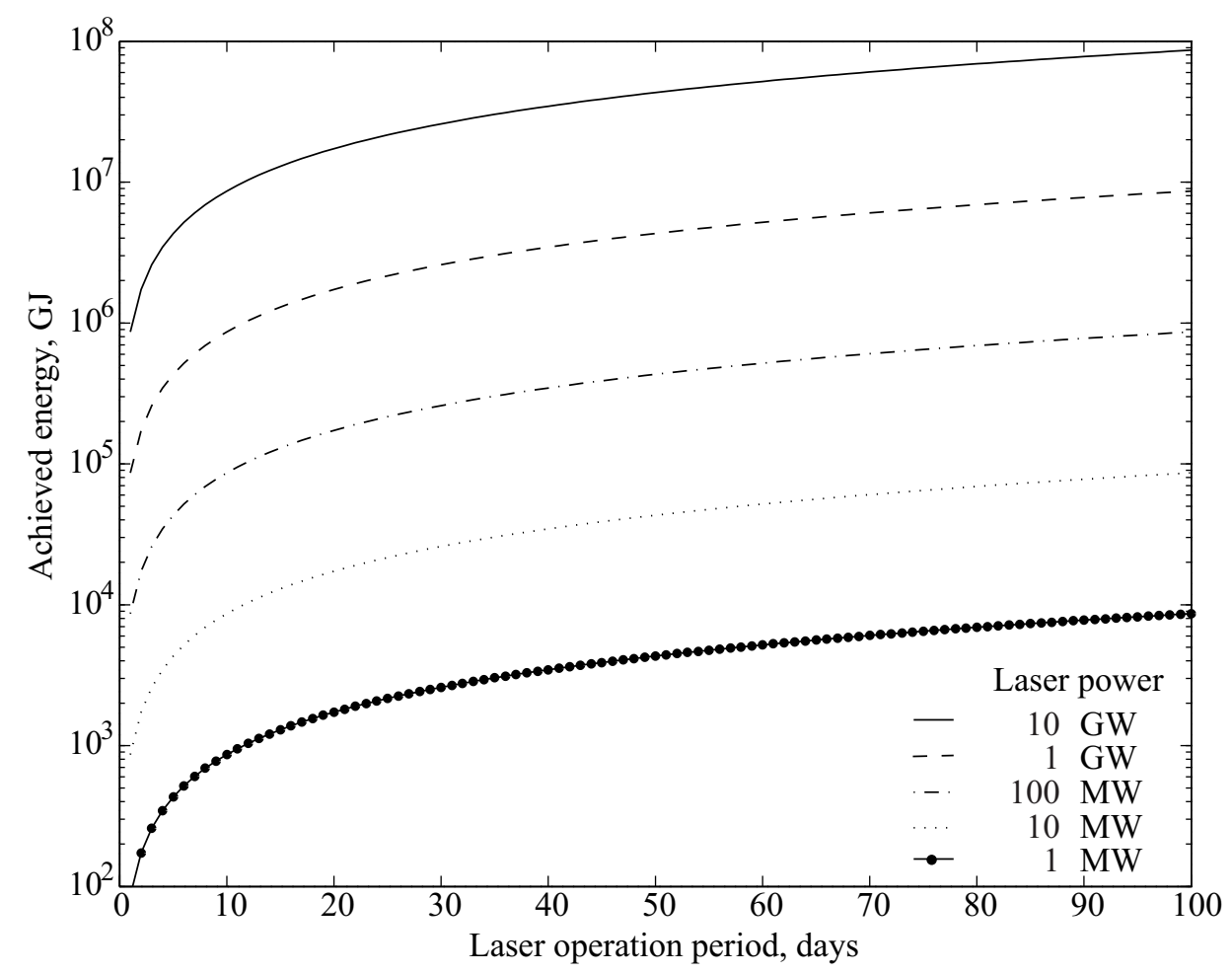

Figure 28. Achieved energy for given laser power and operation period.

continuously for about 3 months beginning 1 year before collision. Although these power levels may not be readily achievable today, they are consistent with the power levels needed for high-thrust, highspecific impulse propulsion systems envisioned for rapid in-space transportation in the future. This power needed for distant rendezvous with an asteroid/comet would then be available for the orbit modification phase of the defense mission.

\section{Overview of Results}

The minimum $\Delta \mathrm{V}$ vector has finer structures associated with the impulse time to deflect an ECO. Both asteroids and comets have similar fluctuation structures of $\Delta \mathrm{V}$ and impulse angle histories with respect to their orbital periods. For a given miss distance and impulse time, the slower Earth-crossing objects relative to the Earth usually require a larger $\Delta \mathrm{V}$. When the gravitational effects of Earth are considered, the minimum $\Delta \mathrm{V}$ is linearly proportional not to the miss distance but the impact parameter, $b_{\mathrm{i}}$. The effects of orbital inclination on $\Delta \mathrm{V}$ and impulse angles are very small for the deflection problem. The $\Delta \mathrm{V}$ and impulse angles are more sensitive to eccentricity and orbital period rather than the inclination. The velocity increment, $\Delta \mathrm{V}_{\mathrm{W}}$, normal to orbital plane of Earth-crossing objects is negligible for most deflection missions. The case of preperihelial impact has slightly larger local minima in the magnitude of impulse than the case of postperihelial impact, and preperihelial impact cases have more fluctuations in optimal impulse angle than postperihelial impact cases. At the final stage, where impulse time approaches impact time, the optimal impulse has been changed dramatically and the $\Delta \mathrm{V}$ is logarithmically increased. Earth-crossing asteroids and comets may have no significant differences in optimal $\Delta \mathrm{V}$ and impulse angles when impulse time is within a few months. 
The final interceptor mass (or energy) also changes according to the fluctuations in the minimum $\Delta \mathrm{V}$ required for the space deflection missions. The optimal deflection strategy is dependent on the size and composition of an asteroid/comet as well as the amount of warning time. The potential of several deflection approaches, including high-thrust engine, kinetic deflection, nuclear detonation, and laser ablation, is discussed. A high-thrust method requires much more mass than other methods. Kinetic-energy deflection needs about a few tens or a few hundreds times less mass than a high-thrust method. Nuclear detonations reduce final payload mass by several orders of magnitude compared with the kinetic-energy deflection method. For a given diameter target, the interceptor mass for comet deflection is usually less than the mass needed for asteroid deflection. Although not as mass or energy efficient as a nuclear detonation or kinetic-energy impact, a laser ablation system has significant advantages and the potential to provide controlled orbit modification of Earth-crossing celestial bodies.

\section{Concluding Remarks}

Deflection or orbit modification of potentially Earth-impacting asteroids/comets has been considered. Several techniques were assessed.

These analyses demonstrate that the optimal deflection strategy is highly dependent on the size and the orbital elements of the asteroid/comet, as well as the amount of warning time. It is concluded that the best deflection strategy and optimal impulse change in velocity $(\Delta \mathrm{V})$ should be carefully investigated if an impacting object is discovered, provided that sufficient time is available. Depending on when the $\Delta \mathrm{V}$ can be applied to an Earth-crossing object (ECO), the optimal impulse angle is not necessarily along the velocity or antivelocity vector, particularly when the impulse time is less than 1 orbit before impact. Although not as mass or energy efficient as a nuclear detonation or kinetic-energy impact, a laser ablation system has significant advantages including the potential to provide controlled orbit modification of ECOs. Regardless of the method employed, early application of the impulse dominates the magnitude of the deflection effort. Impacting objects confirmed years or decades in advance are much easier to deflect and allow a detailed assessment and development of a planetary defense strategy. However, the use of operational space-based elements in the event of a confirmed threat could provide a reliable, rapid response to ECOs, even with relatively short warning times.

\section{References}

1. Ahrens, T. J.; and Harris, A. W.: Deflection and Fragmentation of Near-Earth Asteroids. In Hazards Due to Comets \& Asteroids, T. Gehrels, ed., The University of Arizona Press, 1994, pp. 897-928.

2. Solem, J. C.: Interception of Comets and Asteroids on Collision Course With Earth. J. Spacecr. \& Rockets, vol. 30, no. 2, 1993, pp. 222-228.

3. Ivashkin, V. V.; and Smirnov, V. V.: An Analysis of Some Methods of Asteroid Hazard Mitigation for the Earth. J. Planet. Space Sci., vol. 43, no. 6, 1995, pp. 821-825.

4. Simonenko, V. A.; Nogin, V. N.; Petrov, D. V.; Shubin, O. N.; and Solem, J. C.: Defending the Earth Against Impacts From Large Comets and Asteroids. In Hazards Due to Comets \& Asteroids, T. Gehrels, ed., The University of Arizona Press, 1994, pp. 929-953.

5. Tedeschi, W. J.: Mitigation of the NEO Impact Hazard Using Kinetic Energy. Proceedings of the Planetary Defense Workshop, Lawrence Livermore National Laboratory, Livermore, CA, May 1995, URL: $<$ http://www.llnl.gov/tid/lof/documents/toc /232015.html $>$ [cited 5 Oct. 2001].

6. Park, S.-Y.; and Ross, I. M.: Two-Body Optimization for Deflecting Earth-Crossing Asteroids. J. Guid., Control, \& Dyn., vol. 22, no. 3, 1999, pp. 415-420. 
7. Ross, I. M.; Park, S.-Y.; and Porter, S. D.: Gravitational Effects of Earth in Optimizing Delta-V for Deflecting Earth-Crossing Asteroids. J. Spacecr. \& Rockets, vol. 38, no. 5, Sept.-Oct. 2001, pp. 759-764.

8. Conway, B. A.: Near-Optimal Deflection of Earth-Approaching Asteroids. J. Guid., Control, \& Dyn., vol. 24, no. 5, 2001, pp.1035-1037.

9. Guelman, M.; and Harel, D.: Power Limited Soft Landing on an Asteroid. J. Guid., Control, \& Dyn., vol. 17, no. 1, 1994, pp. 15-20.

10. Remo, J. L.; and Sforza, P. M.: Subsurface Momentum Coupling Analysis for Near-Earth-Object Orbital Management. Acta Astronautica, vol. 35, no. 1, 1995, pp. 27-33.

11. Melosh, H. J.; Nemchinov, I. V.; and Zetzer, Yu. I.: Non-Nuclear Strategies for Deflecting Comets and Asteroids. In Hazards Due to Comets \& Asteroids, T. Gehrels, ed., The University of Arizona Press, 1994, pp. 1111-1132.

12. Meissinger, H. F.: Technology Assessment for Defense Against Asteroids or Comet. Proceedings of the Planetary Defense Workshop, Lawrence Livermore National Laboratory, Livermore, CA, May 1995.

13. Hale, F. J.: Introduction to Space Flight. Prentice Hall, Englewood Cliffs, NJ, 1994, p. 89.

14. Battin, R. H.: An Introduction to the Mathematics and Methods of Astrodynamics. American Institute of Aerodynamics and Astronautics, Inc., New York, 1987, pp. 153-178.

15. Grace, A.: Optimization TOOLBOX User's Guide, The MathWorks, Inc., 1992, pp. 1.13-3.12.

16. Phipps, C. R.: Lasers Can Play an Important Role in the Planetary Defense. Proceedings of the Planetary Defense Workshop, Lawrence Livermore National Laboratory, Livermore, CA, May 1995.

17. Canavan, G. H.; and Solem, J. C.: Near-Earth Object Interception Workshop. In Hazards Due to Comets \& Asteroids, T. Gehrels, ed., The University of Arizona Press, 1994, pp. 93-124.

18. Canavan, G. H.; Solem, J. C.; and Rather, J. D. G., eds.: Proceedings of the Near-Earth-Object Interception Workshop, Los Alamos National Laboratory, Feb. 1993, pp. 202-205.

19. Canavan, G. H.: Cost and Benefits of Near-Earth Object Defenses. Proceedings of the Planetary Defense Workshop, Lawrence Livermore National Laboratory, Livermore, CA, May 1995.

20. Campbell, J. W.: Project ORION: Orbital Debris Removal Using Ground-Based Sensors and Lasers. NASA TM 108522, MSFC, AL, 1996.

21. Campbell, J. W.; and Mazanek, D. D.: Laser Solutions for Reducing the Environment Risks Associated With Orbital Debris and Near Earth Objects. Proceedings of 52nd International Astronautical Congress, IAF-01-C.2.01, 1-5 Oct. 2001, Toulouse, France. 